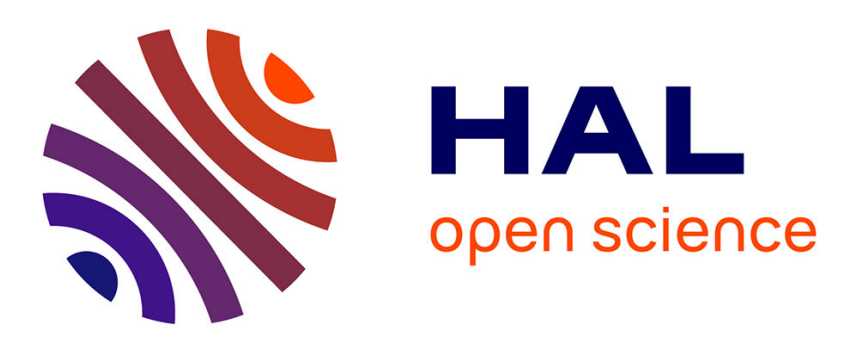

\title{
AN HYPERBOLIC-PARABOLIC PREDATOR-PREY MODEL INVOLVING A VOLE POPULATION STRUCTURED IN AGE
}

Giuseppe Maria M Coclite, Carlotta Donadello, Thi Nhu Thao Nguyen

\section{- To cite this version:}

Giuseppe Maria M Coclite, Carlotta Donadello, Thi Nhu Thao Nguyen. AN HYPERBOLICPARABOLIC PREDATOR-PREY MODEL INVOLVING A VOLE POPULATION STRUCTURED IN AGE. 2021. hal-02936776v2

\section{HAL Id: hal-02936776 \\ https://hal.science/hal-02936776v2}

Preprint submitted on 27 Feb 2021

HAL is a multi-disciplinary open access archive for the deposit and dissemination of scientific research documents, whether they are published or not. The documents may come from teaching and research institutions in France or abroad, or from public or private research centers.
L'archive ouverte pluridisciplinaire HAL, est destinée au dépôt et à la diffusion de documents scientifiques de niveau recherche, publiés ou non, émanant des établissements d'enseignement et de recherche français ou étrangers, des laboratoires publics ou privés. 


\title{
AN HYPERBOLIC-PARABOLIC PREDATOR-PREY MODEL INVOLVING A VOLE POPULATION STRUCTURED IN AGE
}

\author{
G. M. COCLITE, C. DONADELLO, AND T. N. T. NGUYEN
}

\begin{abstract}
We prove existence and stability of entropy solutions for a predator-prey system consisting of an hyperbolic equation for predators and a parabolic-hyperbolic equation for preys. The preys' equation, which represents the evolution of a population of voles as in 2, depends on time, $t$, age, $a$, and on a 2-dimensional space variable $x$, and it is supplemented by a nonlocal boundary condition at $a=0$. The drift term in the predators' equation depends nonlocally on the density of preys and the two equations are also coupled via classical source terms of Lotka-Volterra type, as in [4. We establish existence of solutions by applying the vanishing viscosity method, and we prove stability by a doubling of variables type argument.
\end{abstract}

\section{INTRODUCTION}

1.1. The model and the assumptions. Our goal in this paper is to investigate the wellposedness of a predator-prey model extending the model for a vole population structured in age we introduced in [2]. To this end we couple the latter equation to the hyperbolic equation for predators proposed in [4] in which the drift depends nonlocally on the density of preys, so that the predators tend to move toward the regions in which preys are more abundant. The system we consider writes as follows

$$
\begin{cases}\partial_{t} u+\operatorname{div}_{x}(u \nu(\phi))=(\mathfrak{b}(\phi)-\beta) u, & (t, x) \in(0, T) \times \mathbb{R}^{2}, \\ \partial_{t} \rho+\partial_{a} \rho+\operatorname{div}_{x}\left(\rho \chi_{1}(a) \mathbf{v}(x) Y_{\theta}(\phi-R)\right)=\mu \Delta_{x} \rho-\mathfrak{d}(t, a, x) \rho-\mathfrak{p}(a, u) \rho, & (t, a, x) \in(0, T)^{2} \times \mathbb{R}^{2}, \\ \rho(t, 0, x)=\mathcal{A}(\phi)\left(\int_{0}^{\infty} \rho(t, a, x) \chi_{3}(a) \mathrm{d} a\right) \omega(t, x), & (t, x) \in(0, T) \times \mathbb{R}^{2}, \\ \rho(0, a, x)=\rho_{0}(a, x), & (a, x) \in(0, T) \times \mathbb{R}^{2}, \\ u(0, x)=u_{0}(x), & x \in \mathbb{R}^{2},\end{cases}
$$

where $u=u(t, x)$ and $\phi=\phi(t, x)$ represent the respective density of predators and preys at $(t, x)$. Since the prey population is also structured on age its dynamics is better described by $\rho=\rho(t, a, x)$, which is the density of preys of age $a$ at $(t, x)$. More precisely, the relation between $\phi$ and $\rho$ is given by

$$
\phi(t, x)=\int_{0}^{\infty} \rho(t, a, x) \chi_{2}(a) \mathrm{d} a,
$$

where $\chi_{2}(a)$ is an approximation of the indicator function of the interval $(\sigma, T)$, where $T$ is the target time of our observation and $0<\sigma \ll 1$. The parameter $\sigma$ does not play a role in the modeling, but allows to avoid technical difficulties in our analysis. In the first equation, the function $\mathfrak{b}(\phi)$ represents the reproduction rate of predators depending on preys' availability, while $\beta>0$, the predators' mortality rate, is assumed to be constant. As in [4] the flux of $u$ is driven to the direction of higher preys'

Date: February 19, 2021.

2020 Mathematics Subject Classification. 35Q92, 35M33.

Key words and phrases. population dynamics, predator-prey systems, parabolic-hyperbolic equations, nonlocal conservation laws, nonlocal boundary value problem. 
concentration by a nonlinear, nonlocal velocity $\nu$ of the form

$$
\nu(\phi)=\kappa \frac{\nabla(\phi * \eta)}{\sqrt{1+\|\nabla(\phi * \eta)\|^{2}}},
$$

where $\kappa>0$ is the maximal speed of predators and $\eta$ is a positive smooth mollifier with $\int_{\mathbb{R}^{2}} \eta \mathrm{d} x=1$ so that the convolution $(\phi(t) * \eta)(x)$ represents an average of the density of preys in a neighborhood of $x$ at time $t$.

The equation for the preys, introduced in [2], is related to classical models for the dynamics of a population structured in age, see [3, 10, 13, but the choice of the coefficients and boundary conditions at $a=0$ takes into account the data collections and ecological considerations in [1, 5, 6, 8, 12, We recall here the essential assumptions on the form of the coefficients.

We introduce constants $0<A_{1}<A_{2}$ so that a vole is young (baby) if its age $a$ is in $\left(0, A_{1}\right)$, juvenile if its age is in $\left(A_{1}, A_{2}\right)$ and adult otherwise. The three age classes differ as babies do not reproduce, adults' mortality rate is lower and juveniles exhibit a significant spatial dynamic during dispersals. Dispersal is a characteristic phenomenon of vole populations, correlated to overcrowding. Whenever the density of voles $\phi$ rises above a threshold value $R>0$, representing a fraction of the capacity of the environment, the juvenile individuals leave their original colony and disperse over relatively large distances ( 0.5 to $5 \mathrm{~km}$ ) with velocity $\mathbf{v}(x)$. We fix $\theta>0$ and we consider an approximation of the Heaviside function, $Y_{\theta}$, defined as

$$
Y(\xi)=\left\{\begin{array}{ll}
1, & \text { if } \xi \geq 0, \\
0, & \text { if } \xi \leq-1,
\end{array} \quad Y^{\prime}(\xi) \geq 0, \quad Y_{\theta}(\xi)=Y\left(\frac{\xi}{\theta}\right)\right.
$$

From $Y_{\theta}$ we costruct the approximations of the indicator functions of the intervals $(\sigma, T),\left(A_{1}, A_{2}\right)$, and $\left(A_{1}, T\right)$

$$
\chi_{1}(a)=Y_{\theta}\left(a-A_{1}\right) Y_{\theta}\left(A_{2}-a\right), \quad \chi_{2}(a)=Y_{\theta}(a-\sigma) Y_{\theta}(T-a), \quad \chi_{3}(a)=Y_{\theta}\left(a-A_{1}\right) Y_{\theta}(T-a) .
$$

The mortality rate of voles splits into two terms: $\mathfrak{p}=\mathfrak{p}(a, u)$ represents the mortality due to the presence of the specific predator whose density is $u$, while $\mathfrak{d}=\mathfrak{d}(t, a, x)$ stands for all other casualties (sickness, starvation, generic predation, etc).

The second-order term $\mu \Delta_{x} \rho$ represents short range spatial dynamics related to foraging activities. Everywhere in the following $\theta$ and $\mu>0$ are fixed.

In the boundary condition at $a=0$, the function $\omega=\omega(t, x)$ is the reproduction rate of voles depending on time and position. Both these parameters are significant here, as the beginning and the end of the reproduction season are strongly connected to the average temperature over one week, see [7] and references therein. The function $\mathcal{A}(\phi)$ describes the influence of the total density of voles on natality. Examples of non constant $\mathcal{A}$ are functions of the form

$$
\mathcal{A}(\phi)=\frac{\alpha \phi^{\gamma}}{(\beta+\phi)^{\gamma}}
$$

for different choices of $\alpha, \beta$ and $\gamma$.

The fourth and fifth equations are the respective initial conditions at $t=0$ for voles and predators. 
1.2. Assumptions. The coefficients $\mathfrak{b}, \mathbf{v}, \mathfrak{d}, \mathfrak{p}, \mathcal{A}, \omega$ and the initial data $\rho_{0}, u_{0}$ of system (1.1) satisfy the following conditions:

$$
\begin{aligned}
& \mathfrak{b} \in C^{\infty}(\mathbb{R}) \cap W^{1, \infty}(\mathbb{R}), \quad \mathfrak{b}(\cdot) \geq 0, \\
& \mathbf{v} \in C^{\infty}\left(\mathbb{R}^{2}\right) \cap L^{2}\left(\mathbb{R}^{2}\right) \cap L^{\infty}\left(\mathbb{R}^{2}\right), \quad \operatorname{div}_{x}(\mathbf{v}) \in L^{1}\left(\mathbb{R}^{2}\right) \cap W^{2, \infty}\left(\mathbb{R}^{2}\right), \quad \mathbf{v}>0, \\
& \mathfrak{d} \in C^{\infty}\left([0, \infty) \times[0, \infty) \times \mathbb{R}^{2}\right) \cap W^{2, \infty}\left((0, \infty) \times(0, \infty) \times \mathbb{R}^{2}\right), \quad 0<d_{*} \leq \mathfrak{d}(\cdot, \cdot, \cdot) \leq d^{*}, \\
& \mathfrak{p} \in C^{\infty}([0, \infty) \times \mathbb{R}) \cap W^{2, \infty}((0, \infty) \times \mathbb{R}), \quad 0<p_{*} \leq \mathfrak{p}(\cdot, \cdot) \leq p^{*}, \\
& \mathcal{A} \in C^{\infty}(\mathbb{R}) \cap L^{\infty}(\mathbb{R}), \quad \mathcal{A}(\cdot) \geq 0, \quad \mathcal{A}(0)=0, \quad\left|\mathcal{A}^{\prime}(\xi) \xi\right|,\left|\mathcal{A}^{\prime \prime}(\xi) \xi\right| \leq C_{0}, \\
& \omega \in C^{\infty}\left([0, \infty) \times \mathbb{R}^{2}\right) \cap W^{1, \infty}\left((0, \infty) \times \mathbb{R}^{2}\right), \quad \omega(\cdot, \cdot) \geq 0, \\
& \rho_{0} \in L^{1}\left((0, \infty) \times \mathbb{R}^{2}\right) \cap L^{\infty}\left((0, \infty) \times \mathbb{R}^{2}\right), \quad \rho_{0} \geq 0, \\
& \sup _{x \in \mathbb{R}^{2}}\left\|\rho_{0}(\cdot, x)\right\|_{L^{1}(0, \infty)}, \sup _{a>0}\left\|\rho_{0}(a, \cdot)\right\|_{L^{1}\left(\mathbb{R}^{2}\right)}, \int_{\mathbb{R}^{2}} T V\left(\rho_{0}(\cdot, x)\right) \mathrm{d} x \leq C_{0}, \\
& u_{0} \in L^{1}\left(\mathbb{R}^{2}\right) \cap B V\left(\mathbb{R}^{2}\right) \cap L^{\infty}\left(\mathbb{R}^{2}\right), \quad u_{0} \geq 0,
\end{aligned}
$$

for some positive constants $d_{*}, d^{*}, p_{*}, p^{*}, C_{0}$.

For what the velocity of predators, $\nu$, is concerned, we assume that the mollifier $\eta$ satisfies

$$
\nabla_{x} \eta \in\left(C^{2} \cap W^{2,2} \cap W^{1, \infty}\right)\left(\mathbb{R}^{2}, \mathbb{R}^{2}\right) .
$$

1.3. Main result. Our main result is the wellposedness of entropy weak solutions for system (1.1), stated in Theorem 1.1. We adopt the following definitions of weak solution and entropy solution.

Definition 1.1. We say that the pair $(u, \rho)$ is a weak solution of (1.1) if the following holds for every $T>0$.

(D.1) $\rho \geq 0, \rho \in L^{\infty}\left(0, T ; L^{1}\left((0, \infty) \times \mathbb{R}^{2}\right)\right) \cap L^{\infty}\left((0, T) \times(0, \infty) \times \mathbb{R}^{2}\right) \cap L^{2}\left((0, T) \times(0, \infty) ; H^{2}\left(\mathbb{R}^{2}\right)\right)$.

(D.2) $u \in L^{1}\left((0, T) \times \mathbb{R}^{2}\right) \cap B V\left((0, T) \times \mathbb{R}^{2}\right)$.

(D.3) For almost every $(t, x) \in(0, T) \times \mathbb{R}^{2}, \rho(t, \cdot, x) \in B V(0, \infty)$ and

$$
\rho\left(t, 0^{+}, x\right)=\mathcal{A}(\phi)\left(\int_{0}^{\infty} \rho(t, a, x) \chi_{3}(a) d a\right) \omega(t, x),
$$

where $\rho\left(t, 0^{+}, x\right)$ is the trace of $\rho(t, \cdot, x)$ at $a=0$.

def:4 (D.4) For every test function $\xi \in C_{c}^{\infty}\left(\mathbb{R}^{4}\right)$

$$
\begin{aligned}
& \int_{0}^{\infty} \int_{0}^{\infty} \int_{\mathbb{R}^{2}}\left(\rho \partial_{t} \xi+\rho \partial_{a} \xi+\rho \chi_{1}(a) \mathbf{v} \cdot \nabla_{x} \xi Y_{\theta}(\phi-R)+\mu \rho \Delta_{x} \xi-\mathfrak{d} \rho \xi-\mathfrak{p} \rho \xi\right) d x d a d t \\
& \quad+\int_{0}^{\infty} \int_{0}^{\infty} \int_{\mathbb{R}^{2}} \mathcal{A}(\phi) \rho(t, a, x) \chi_{3}(a) \omega(t, x) \xi(t, 0, x) d x d a d t \\
& \quad+\int_{0}^{\infty} \int_{\mathbb{R}^{2}} \rho_{0}(a, x) \xi(0, a, x) d x d a=0, \\
& \int_{0}^{\infty} \int_{0}^{\infty} \int_{\mathbb{R}^{2}}\left(u \partial_{t} \xi+u \nu(\phi) \cdot \nabla_{x} \xi+(\mathfrak{b}(\phi)-\beta) u \xi\right) d x d a d t+\int_{0}^{\infty} \int_{\mathbb{R}^{2}} u_{0}(x) \xi(0, a, x) d x d a=0 .
\end{aligned}
$$


Definition 1.2. We say that a weak solution $(u, \rho)$ is an entropy solution of (1.1) if for any nonnegative test function $\xi \in C^{\infty}\left(\mathbb{R}^{4}\right)$ with compact support and for any constant $\mathfrak{c} \in \mathbb{R}$ there hold

$$
\begin{aligned}
& \int_{0}^{\infty} \int_{0}^{\infty} \int_{\mathbb{R}^{2}}\left(|\rho-\mathfrak{c}|\left(\partial_{t} \xi+\partial_{a} \xi\right)-\operatorname{div}_{x}\left(|\rho-\mathfrak{c}| \chi_{1} \mathbf{v} Y_{\theta}(\phi-R)\right) \xi\right. \\
& \left.\quad+\mu \Delta_{x}|\rho-\mathfrak{c}| \xi-\operatorname{sign}(\rho-\mathfrak{c})(\mathfrak{d}+\mathfrak{p}) \rho \xi\right) d x d a d t \\
& \quad+\int_{0}^{\infty} \int_{\mathbb{R}^{2}}\left|\rho\left(t, 0^{+}, x\right)-\mathfrak{c}\right| \xi(t, 0, x) d x d t \\
& \quad+\int_{0}^{\infty} \int_{\mathbb{R}^{2}}\left|\rho_{0}(a, x)-\mathfrak{c}\right| \xi(0, a, x) d x d a \\
& \geq \int_{0}^{\infty} \int_{0}^{\infty} \int_{\mathbb{R}^{2}} \mathfrak{c} \operatorname{sign}(\rho-\mathfrak{c}) \chi_{1}(a) \operatorname{div}_{x}\left(\mathbf{v}(x) Y_{\theta}(\phi-R)\right) \xi d x d a d t
\end{aligned}
$$

and

$$
\begin{aligned}
\int_{0}^{\infty} & \int_{0}^{\infty} \int_{\mathbb{R}^{2}}\left(|u-\mathfrak{c}| \partial_{t} \xi+|u-\mathfrak{c}| \nu(\phi) \cdot \nabla_{x} \xi+\operatorname{sign}(u-\mathfrak{c})(\mathfrak{b}(\phi)-\beta) u \xi\right) d x d a d t \\
& +\int_{0}^{\infty} \int_{\mathbb{R}^{2}}\left|u_{0}(x)-\mathfrak{c}\right| \xi(0, a, x) d x d a \geq \int_{0}^{\infty} \int_{0}^{\infty} \int_{\mathbb{R}^{2}} \mathfrak{c} \operatorname{sign}(u-\mathfrak{c}) \operatorname{div}_{x}(\nu(\phi)) \xi d x d a d t
\end{aligned}
$$

Theorem 1.1. Assume (1.4)-2.4), then the initial boundary value problem (1.1) admits a unique entropy solution $(u, \rho)$ in the sense of Definition 1.2 . Moreover, if $\left(u_{1}, \rho_{1}\right)$ and $\left(u_{2}, \rho_{2}\right)$ are the two entropy solutions of (1.1) having initial data $\left(u_{1,0}, \rho_{1,0}\right)$ and $\left(u_{2,0}, \rho_{2,0}\right)$, then there exists a positive constant $C$ such that the following estimate holds for almost every $t \geq 0$

$$
\begin{aligned}
\left\|u_{1}(t, \cdot)-u_{2}(t, \cdot)\right\|_{L^{1}\left(\mathbb{R}^{2}\right)}+ & \left\|\rho_{1}(t, \cdot, \cdot)-\rho_{2}(t, \cdot, \cdot)\right\|_{L^{1}\left((0, \infty) \times \mathbb{R}^{2}\right)} \\
& \leq C e^{C e^{C t}}\left\|\rho_{1,0}-\rho_{2,0}\right\|_{L^{1}\left((0, \infty) \times \mathbb{R}^{2}\right)}+C e^{C e^{C t}}\left\|u_{1,0}-u_{2,0}\right\|_{L^{1}\left(\left(\mathbb{R}^{2}\right)\right.}
\end{aligned}
$$

The fixed point argument used in [4] to prove existence and stability for a predator-prey system does not apply to our system in a straightforward way because it requires extremely fine information on the coefficients appearing in the a priori estimates for both predators' and preys' equations. This is not easy to obtain in our case, as the equation we use for preys comes from a specific population model and its analytical study is rather technical.

In Section 2 we introduce a sequence of parabolic approximations of problem (1.1), for which we prove suitable a priori estimates. Then we apply the compensated compactness lemma by Panov, see [11, to show the strong compactness of the sequence for voles, while the strong convergence of the sequence of predators is ensured by Helly's theorem. Lemma 2.14 establishes the existence of an entropy solution in the sense of Definition 1.2. The uniqueness and stability of such solutions are proved in Section 3 using a doubling of variables type argument.

\section{EXISTENCE}

The existence argument is based on the compactness analysis of a sequence of solutions to approximating problems defined as follows. For any given $\varepsilon>0$, we let $\left(\rho_{\varepsilon}=\rho_{\varepsilon}(t, a, x), u_{\varepsilon}=u_{\varepsilon}(t, x)\right)$ be a 
solution of the problem

$$
\begin{cases}\partial_{t} u_{\varepsilon}+\operatorname{div}_{x}\left(u_{\varepsilon} \nu\left(\phi_{\varepsilon}\right)\right)=\left(\mathfrak{b}\left(\phi_{\varepsilon}\right)-\beta\right) u_{\varepsilon}+\varepsilon \Delta_{x} u_{\varepsilon}, & (t, x) \in(0, T) \times \mathbb{R}^{2}, \\ \partial_{t} \rho_{\varepsilon}+\partial_{a} \rho_{\varepsilon}+\operatorname{div}_{x}\left(\rho_{\varepsilon} \chi_{1}(a) \mathbf{v}(x) Y_{\theta}\left(\phi_{\varepsilon}-R\right)\right) & \\ \quad=\mu \Delta_{x} \rho_{\varepsilon}-\mathfrak{d}(t, a, x) \rho_{\varepsilon}-\mathfrak{p}\left(a, u_{\varepsilon}\right) \rho_{\varepsilon}+\varepsilon \partial_{a}\left(\chi(a) \partial_{a} \rho_{\varepsilon}\right), & (t, a, x) \in(0, T)^{2} \times \mathbb{R}^{2}, \\ \rho_{\varepsilon}(t, 0, x)=\mathcal{A}\left(\left|\phi_{\varepsilon}\right|\right)\left(\int_{0}^{\infty}\left|\rho_{\varepsilon}(t, a, x)\right| \chi_{3}(a) \mathrm{d} a\right) \omega(t, x), & (t, x) \in(0, T) \times \mathbb{R}^{2}, \\ \rho_{\varepsilon}(0, a, x)=\rho_{0, \varepsilon}(a, x), & (a, x) \in(0, T)^{2} \times \mathbb{R}^{2}, \\ u_{\varepsilon}(0, x)=u_{0, \varepsilon}(x), & x \in \mathbb{R}^{2},\end{cases}
$$

where

$$
\phi_{\varepsilon}(t, x)=\int_{0}^{\infty} \rho_{\varepsilon}(t, a, x) \chi_{2}(a) \mathrm{d} a,
$$

$\chi(a) \in C^{\infty}([0,+\infty),[0,1])$ satisfies $\chi(0)=0$, and $\left\{\left(\rho_{0, \varepsilon}, u_{0, \varepsilon}\right)\right\}_{\varepsilon}$ is a family of approximations of the initial condition $\left(\rho_{0}, u_{0}\right)$ such that

$$
\begin{array}{ll}
\rho_{0, \varepsilon} \in C^{\infty}\left((0, \infty) \times \mathbb{R}^{2}\right), u_{0, \varepsilon} \in C^{\infty}\left(\mathbb{R}^{2}\right), & \varepsilon>0, \\
\rho_{0, \varepsilon} \rightarrow \rho_{0}, \quad \text { a.e. and in } L^{p}\left((0, \infty) \times \mathbb{R}^{2}\right), 1 \leq p<\infty, \text { as } \varepsilon \rightarrow 0, & \\
u_{0, \varepsilon} \rightarrow u_{0}, \quad \text { a.e. and in } L^{p}\left(\mathbb{R}^{2}\right), 1 \leq p<\infty, \text { as } \varepsilon \rightarrow 0, & \\
\rho_{0, \varepsilon} \geq 0,\left\|\rho_{0, \varepsilon}\right\|_{L^{1}\left((0, \infty) \times \mathbb{R}^{2}\right)} \leq C, & \varepsilon \geq 0, \\
\sup _{x \in \mathbb{R}^{2}}\left\|\rho_{0, \varepsilon}(\cdot, x)\right\|_{L^{1}(\mathbb{R})}, \sup _{a \geq 0}\left\|\rho_{0, \varepsilon}(a, \cdot)\right\|_{L^{1}\left(\mathbb{R}^{2}\right)},\left\|\partial_{a} \rho_{0, \varepsilon}\right\|_{L^{1}\left((0, \infty) \times \mathbb{R}^{2}\right)} \leq C, & \varepsilon \geq 0, \\
\left\|u_{0, \varepsilon}\right\|_{L^{1}\left(\mathbb{R}^{2}\right)},\left\|\nabla_{x} u_{0, \varepsilon}\right\|_{L^{1}\left(\mathbb{R}^{2}\right)}, \varepsilon\left\|\Delta_{x} u_{0, \varepsilon}\right\|_{L^{1}\left(\mathbb{R}^{2}\right)} \leq C, u_{0, \varepsilon} \geq 0, & \varepsilon>0 .
\end{array}
$$

2.1. A priori estimates. In this section we establish the a priori estimates on $\left(u_{\varepsilon}, \rho_{\varepsilon}\right)$ which are necessary to pass to the limit as $\varepsilon \rightarrow 0$ in 2.1.

From now on we use the notation $C$ for all the positive constants independent on $\varepsilon$ appearing in the text or in the statements of our results, while in proofs we write $c$ to indicate any positive constant non depending on $\varepsilon$, and $c_{T}$ for quantities of the form $c e^{c t}, t \in(0, T)$. The proof of the following preliminary Lemma is postposed to Section 4 (see also [4, Lemma 4.1]).

Lemma 2.1. Let $\eta$ be such that

$$
\nabla_{x} \eta \in\left(C^{2} \cap W^{2,2} \cap W^{1, \infty}\right)\left(\mathbb{R}^{2}, \mathbb{R}^{2}\right) .
$$

Then the map $\nu: L^{1}\left(\mathbb{R}^{2} ; \mathbb{R}\right) \mapsto C^{\infty}(\mathbb{R}) \cap W^{1, \infty}\left(\mathbb{R}^{2} ; \mathbb{R}^{2}\right)$ satisfies

ass : nu1

ass : nu2

ass : nu3

ass : nu4

ass:nu5

ass : nu6

$$
\begin{aligned}
& \|\nu(\phi)\|_{L^{\infty}\left(\mathbb{R}^{2} ; \mathbb{R}^{2}\right)} \leq K\|\phi\|_{L^{1}\left(\mathbb{R}^{2}, \mathbb{R}\right)} \\
& \left\|\operatorname{div}_{x}(\nu(\phi))\right\|_{L^{2}\left(\mathbb{R}^{2}, \mathbb{R}\right)} \leq K\|\phi\|_{L^{1}\left(\mathbb{R}^{2} ; \mathbb{R}\right)}, \\
& \left\|\nabla_{x} \nu(\phi)\right\|_{L^{\infty}\left(\mathbb{R}^{2}, \mathbb{R}^{2 \times 2}\right)} \leq K\|\phi\|_{L^{1}\left(\mathbb{R}^{2} ; \mathbb{R}\right)} \\
& \left\|\nu\left(\phi_{1}\right)-\nu\left(\phi_{2}\right)\right\|_{L^{\infty}\left(\mathbb{R}^{2}, \mathbb{R}^{2}\right)} \leq K\left\|\phi_{1}-\phi_{2}\right\|_{L^{1}\left(\mathbb{R}^{2}, \mathbb{R}\right)}, \\
& \left\|\nabla_{x}\left(\operatorname{div}_{x}(\nu(\phi))\right)\right\|_{L^{2}\left(\mathbb{R}^{2}, \mathbb{R}^{2}\right)} \leq K\left(1+K\|\phi\|_{L^{1}\left(\mathbb{R}^{2}, \mathbb{R}\right)}\right)\|\phi\|_{L^{1}\left(\mathbb{R}^{2}, \mathbb{R}\right)}, \\
& \left\|\operatorname{div}_{x}\left(\nu\left(\phi_{1}\right)-\nu\left(\phi_{2}\right)\right)\right\|_{L^{2}\left(\mathbb{R}^{2}, \mathbb{R}\right)} \leq K\left(1+K\left\|\phi_{2}\right\|_{L^{1}\left(\mathbb{R}^{2}, \mathbb{R}\right)}\right)\left\|\phi_{1}-\phi_{2}\right\|_{L^{1}\left(\mathbb{R}^{2}, \mathbb{R}\right)},
\end{aligned}
$$

where $K$ is a positive constant. 
Lemma 2.2 (Nonnegativity of $\rho_{\varepsilon}, \phi_{\varepsilon}, u_{\varepsilon}$ ). We have that

$$
\rho_{\varepsilon} \geq 0, \quad \phi_{\varepsilon} \geq 0, \quad u_{\varepsilon} \geq 0 .
$$

Proof. Consider the function

$$
x \mapsto \eta(x)=-x \mathbb{1}_{(-\infty, 0)}(x) .
$$

and observe that

$$
\eta^{\prime}(x)=-\mathbb{1}_{(-\infty, 0)}(x), \quad \eta(x)=x \eta^{\prime}(x) .
$$

From 2.1 we obtain

$$
\begin{aligned}
& \frac{d}{d t} \int_{0}^{\infty} \int_{\mathbb{R}^{2}} \eta\left(\rho_{\varepsilon}\right) \mathrm{d} x \mathrm{~d} a=\int_{0}^{\infty} \int_{\mathbb{R}^{2}} \eta^{\prime}\left(\rho_{\varepsilon}\right) \partial_{t} \rho_{\varepsilon} \mathrm{d} x \mathrm{~d} a \\
& =-\int_{0}^{\infty} \int_{\mathbb{R}^{2}} \eta^{\prime}\left(\rho_{\varepsilon}\right) \partial_{a} \rho_{\varepsilon} \mathrm{d} x \mathrm{~d} a-\int_{0}^{\infty} \int_{\mathbb{R}^{2}} \operatorname{div}_{x}\left(\rho_{\varepsilon} \chi_{1} \mathbf{v} Y_{\theta}\right) \eta^{\prime}\left(\rho_{\varepsilon}\right) \mathrm{d} x \mathrm{~d} a \\
& +\mu \int_{0}^{\infty} \int_{\mathbb{R}^{2}} \eta^{\prime}\left(\rho_{\varepsilon}\right) \Delta_{x} \rho_{\varepsilon} \mathrm{d} x \mathrm{~d} a-\int_{0}^{\infty} \int_{\mathbb{R}^{2}} \eta^{\prime}\left(\rho_{\varepsilon}\right)(\mathfrak{d}+\mathfrak{p}) \rho_{\varepsilon} \mathrm{d} x \mathrm{~d} a \\
& +\varepsilon \int_{0}^{\infty} \int_{\mathbb{R}^{2}} \eta^{\prime}\left(\rho_{\varepsilon}\right) \partial_{a}\left(\chi(a) \partial_{a} \rho_{\varepsilon}\right) \rho_{\varepsilon} \mathrm{d} x \mathrm{~d} a \\
& =\int_{\mathbb{R}^{2}} \underbrace{\eta\left(\rho_{\varepsilon}(t, 0, x)\right)}_{=0} \mathrm{~d} x+\underbrace{\int_{0}^{\infty} \int_{\mathbb{R}^{2}} \rho_{\varepsilon} \chi_{1}\left(\mathbf{v} \cdot \nabla_{x} \rho_{\varepsilon}\right) Y_{\theta} \eta^{\prime \prime}\left(\rho_{\varepsilon}\right) \mathrm{d} x \mathrm{~d} a}_{=0} \\
& \underbrace{-\mu \int_{0}^{\infty} \int_{\mathbb{R}^{2}} \eta^{\prime \prime}\left(\rho_{\varepsilon}\right)\left(\nabla_{x} \rho_{\varepsilon}\right)^{2} \mathrm{~d} x \mathrm{~d} a}_{\leq 0} \underbrace{-\int_{0}^{\infty} \int_{\mathbb{R}^{2}}(\mathfrak{d}+\mathfrak{p}) \eta\left(\rho_{\varepsilon}\right) \mathrm{d} x \mathrm{~d} a}_{\leq 0} \\
& +\varepsilon \int_{0}^{\infty} \int_{\mathbb{R}^{2}} \partial_{a}\left(\eta^{\prime}\left(\rho_{\varepsilon}\right) \chi(a) \partial_{a} \rho_{\varepsilon}\right) \mathrm{d} x \mathrm{~d} a \underbrace{-\varepsilon \int_{0}^{\infty} \int_{\mathbb{R}^{2}} \eta^{\prime \prime}\left(\rho_{\varepsilon}\right) \chi(a)\left(\partial_{a} \rho_{\varepsilon}\right)^{2}}_{\leq 0} \mathrm{~d} x \mathrm{~d} a \\
& \leq-\varepsilon \int_{\mathbb{R}^{2}} \underbrace{\eta^{\prime}\left(\rho_{\varepsilon}(t, 0, x)\right)}_{=0} \underbrace{\chi(0)}_{=0} \partial_{a} \rho_{\varepsilon}(t, 0, x) \mathrm{d} x=0 .
\end{aligned}
$$

Thus, integrating on $(0, t)$ we obtain $\eta\left(\rho_{\varepsilon}(t, a, x)\right)=0$ which implies that $\rho_{\varepsilon} \geq 0$, and then $\phi_{\varepsilon} \geq 0$.

From (2.1) we obtain

$$
\begin{aligned}
& \frac{d}{d t} \int_{\mathbb{R}^{2}} \eta\left(u_{\varepsilon}\right) \mathrm{d} x=\int_{\mathbb{R}^{2}} \eta^{\prime}\left(u_{\varepsilon}\right) \partial_{t} u_{\varepsilon} \mathrm{d} x \\
& \quad=-\underbrace{\int_{\mathbb{R}^{2}} \eta^{\prime}\left(u_{\varepsilon}\right) \operatorname{div}_{x}\left(u_{\varepsilon} \nu\left(\phi_{\varepsilon}\right)\right) \mathrm{d} x}_{=0}+\int_{\mathbb{R}^{2}} \eta^{\prime}\left(u_{\varepsilon}\right) \underbrace{(\mathfrak{b}(\phi)-\beta)}_{\leq c} u_{\varepsilon} \mathrm{d} x+\underbrace{\varepsilon \int_{\mathbb{R}^{2}} \eta^{\prime}\left(u_{\varepsilon}\right) \Delta_{x} u_{\varepsilon} \mathrm{d} x}_{\leq 0} \\
& \quad \leq c \int_{\mathbb{R}^{2}} \eta\left(u_{\varepsilon}\right) \mathrm{d} x .
\end{aligned}
$$

Integrating on $(0, t)$ and applying Gronwall Lemma we obtain $\eta\left(u_{\varepsilon}(t, x)\right)=0$, so that $u_{\varepsilon} \geq 0$.

Remark 2.1. We can remove the absolute value in the boundary condition for $\rho_{\varepsilon}$ in 2.1.

Lemma $2.3\left(L^{1}\right.$ estimate on $\left.\rho_{\varepsilon}\right)$. For all $t \geq 0$, we have that

$$
\left\|\rho_{\varepsilon}(t, \cdot, \cdot)\right\|_{L^{1}\left((0, \infty) \times \mathbb{R}^{2}\right)} \leq e^{C t} C .
$$


Proof. Due to the nonnegativity of $\rho_{\varepsilon}$ and the boundedness of $\mathfrak{d}, \mathfrak{p}$ we have

$$
\begin{aligned}
\frac{d}{d t} \int_{0}^{\infty} & \int_{\mathbb{R}^{2}}\left|\rho_{\varepsilon}\right| \mathrm{d} x \mathrm{~d} a=\frac{d}{d t} \int_{0}^{\infty} \int_{\mathbb{R}^{2}} \rho_{\varepsilon} \mathrm{d} x \mathrm{~d} a \\
= & -\int_{0}^{\infty} \int_{\mathbb{R}^{2}} \partial_{a} \rho_{\varepsilon} \mathrm{d} x \mathrm{~d} a-\underbrace{\int_{0}^{\infty} \int_{\mathbb{R}^{2}} \operatorname{div}_{x}\left(\rho_{\varepsilon} \chi_{1} \mathbf{v} Y_{\theta}\right) \mathrm{d} x \mathrm{~d} a+\mu \int_{0}^{\infty} \int_{\mathbb{R}^{2}} \Delta_{x} \rho_{\varepsilon} \mathrm{d} x \mathrm{~d} a}_{=0} \\
& -\int_{0}^{\infty} \int_{\mathbb{R}^{2}} \rho_{\varepsilon} \underbrace{(\mathfrak{d}+\mathfrak{p})}_{\leq c} \mathrm{~d} x \mathrm{~d} a+\varepsilon \underbrace{\int_{0}^{\infty} \int_{\mathbb{R}^{2}} \partial_{a}\left(\chi(a) \partial_{a} \rho_{\varepsilon}\right) \mathrm{d} x \mathrm{~d} a}_{=0} \\
\leq & \int_{\mathbb{R}^{2}} \rho_{\varepsilon}(t, 0, x) \mathrm{d} x+c \int_{0}^{\infty} \int_{\mathbb{R}^{2}} \rho_{\varepsilon} \mathrm{d} x \mathrm{~d} a \\
= & \int_{\mathbb{R}^{2}} \mathcal{A}\left(\phi_{\varepsilon}\right)\left(\int_{0}^{\infty} \rho_{\varepsilon}(t, a, x) \chi_{3}(a) \mathrm{d} a\right) \omega(t, x) \mathrm{d} x+c \int_{0}^{\infty} \int_{\mathbb{R}^{2}} \rho_{\varepsilon} \mathrm{d} x \mathrm{~d} a \\
\leq & c \int_{0}^{\infty} \int_{\mathbb{R}^{2}} \rho_{\varepsilon} \mathrm{d} x \mathrm{~d} a .
\end{aligned}
$$

Integrating on $(0, t)$ we get 2.12 thanks to the Gronwall Lemma and the assumptions in 2.3 .

em:L2 rho Lemma $2.4\left(L^{2}\right.$ estimate on $\left.\rho_{\varepsilon}\right)$. For any $t \geq 0$, we have

$$
\left\|\rho_{\varepsilon}(t, \cdot, \cdot)\right\|_{L^{2}\left((0, \infty) \times \mathbb{R}^{2}\right)},\left\|\nabla_{x} \rho_{\varepsilon}\right\|_{L^{2}\left((0, t) \times(0, \infty) \times \mathbb{R}^{2}\right)}, \sqrt{\varepsilon}\left\|\chi \partial_{a} \rho_{\varepsilon}\right\|_{L^{2}\left((0, t) \times(0, \infty) \times \mathbb{R}^{2}\right)} \leq e^{C t} C .
$$

Proof. We recall the equation of $\rho_{\varepsilon}$

$$
\begin{aligned}
& \partial_{t} \rho_{\varepsilon}+\partial_{a} \rho_{\varepsilon}+\operatorname{div}_{x}\left(\rho_{\varepsilon} \chi_{1}(a) \mathbf{v}(x) Y_{\theta}\left(\phi_{\varepsilon}-R\right)\right) \\
& \quad=\mu \Delta_{x} \rho_{\varepsilon}-\mathfrak{d}(t, a, x) \rho_{\varepsilon}-\mathfrak{p}\left(a, u_{\varepsilon}\right) \rho_{\varepsilon}+\varepsilon \partial_{a}\left(\chi(a) \partial_{a} \rho_{\varepsilon}\right) .
\end{aligned}
$$

We multiply (2.14) by $\rho_{\varepsilon}$ and have

$$
\begin{aligned}
\frac{d}{d t} \int_{0}^{\infty} & \int_{\mathbb{R}^{2}} \frac{\rho_{\varepsilon}^{2}}{2} \mathrm{~d} x \mathrm{~d} a=\int_{0}^{\infty} \int_{\mathbb{R}^{2}} \rho_{\varepsilon} \partial_{t} \rho_{\varepsilon} \mathrm{d} x \mathrm{~d} a \\
= & -\int_{0}^{\infty} \int_{\mathbb{R}^{2}} \rho_{\varepsilon} \partial_{a} \rho_{\varepsilon} \mathrm{d} x \mathrm{~d} a-\int_{0}^{\infty} \int_{\mathbb{R}^{2}} \rho_{\varepsilon} \operatorname{div}_{x}\left(\rho_{\varepsilon} \chi_{1} \mathbf{v} Y_{\theta}\right) \mathrm{d} x \mathrm{~d} a+\mu \int_{0}^{\infty} \int_{\mathbb{R}^{2}} \rho_{\varepsilon} \Delta_{x} \rho_{\varepsilon} \mathrm{d} x \mathrm{~d} a \\
& -\int_{0}^{\infty} \int_{\mathbb{R}^{2}} \rho_{\varepsilon}^{2} \underbrace{(\mathfrak{d}+\mathfrak{p})}_{\leq c} \mathrm{~d} x \mathrm{~d} a+\varepsilon \int_{0}^{\infty} \int_{\mathbb{R}^{2}} \rho_{\varepsilon} \partial_{a}\left(\chi(a) \partial_{a} \rho_{\varepsilon}\right) \mathrm{d} x \mathrm{~d} a \\
\leq & \int_{\mathbb{R}^{2}} \frac{\rho_{\varepsilon}(t, 0, x)^{2}}{2} \mathrm{~d} x-\frac{\mu}{2} \int_{0}^{\infty} \int_{\mathbb{R}^{2}}\left|\nabla_{x} \rho_{\varepsilon}\right|^{2} \mathrm{~d} x \mathrm{~d} a+c \int_{0}^{\infty} \int_{\mathbb{R}^{2}}\left(\rho_{\varepsilon} \chi_{1} \mathbf{v} Y_{\theta}\right)^{2} \mathrm{~d} x \mathrm{~d} a \\
& +c \int_{0}^{\infty} \int_{\mathbb{R}^{2}} \rho_{\varepsilon}^{2} \mathrm{~d} x \mathrm{~d} a-\varepsilon \int_{0}^{\infty} \int_{\mathbb{R}^{2}} \chi(a)\left(\partial_{a} \rho_{\varepsilon}\right)^{2} \mathrm{~d} x \mathrm{~d} a \\
\leq & \frac{1}{2} \int_{\mathbb{R}^{2}} \mathcal{A}^{2}\left(\phi_{\varepsilon}\right)\left(\int_{0}^{\infty} \rho_{\varepsilon}(t, a, x) \chi_{3}(a) \mathrm{d} a\right)^{2} \omega^{2}(t, x) \mathrm{d} x+c \int_{0}^{\infty} \int_{\mathbb{R}^{2}} \rho_{\varepsilon}^{2} \mathrm{~d} x \mathrm{~d} a \\
& -\frac{\mu}{2} \int_{0}^{\infty} \int_{\mathbb{R}^{2}}\left|\nabla_{x} \rho_{\varepsilon}\right|^{2} \mathrm{~d} x \mathrm{~d} a-\varepsilon \int_{0}^{\infty} \int_{\mathbb{R}^{2}} \chi(a)\left(\partial_{a} \rho_{\varepsilon}\right)^{2} \mathrm{~d} x \mathrm{~d} a \\
\leq & c \int_{0}^{\infty} \int_{\mathbb{R}^{2}} \rho_{\varepsilon}^{2} \mathrm{~d} x \mathrm{~d} a-\frac{\mu}{2} \int_{0}^{\infty} \int_{\mathbb{R}^{2}}\left|\nabla_{x} \rho_{\varepsilon}\right|^{2} \mathrm{~d} x \mathrm{~d} a-\varepsilon \int_{0}^{\infty} \int_{\mathbb{R}^{2}} \chi(a)\left(\partial_{a} \rho_{\varepsilon}\right)^{2} \mathrm{~d} x \mathrm{~d} a .
\end{aligned}
$$

Integrating over $(0, t)$ and using the Gronwall Lemma we gain

$$
\left\|\rho_{\varepsilon}(t, \cdot, \cdot)\right\|_{L^{2}\left((0, \infty) \times \mathbb{R}^{2}\right)}^{2}+e^{c t} \mu \int_{0}^{t} \int_{0}^{\infty} \int_{\mathbb{R}^{2}} e^{-c s}\left|\nabla_{x} \rho_{\varepsilon}\right|^{2} \mathrm{~d} x \mathrm{~d} a \mathrm{~d} s
$$




$$
+e^{c t} 2 \varepsilon \int_{0}^{t} \int_{0}^{\infty} \int_{\mathbb{R}^{2}} e^{-c s} \chi(a)\left(\partial_{a} \rho_{\varepsilon}\right)^{2} \mathrm{~d} x \mathrm{~d} a \mathrm{~d} s \leq e^{c t}\left\|\rho_{0, \varepsilon}(\cdot, \cdot)\right\|_{L^{2}\left((0, \infty) \times \mathbb{R}^{2}\right)}^{2} .
$$

We obtain (2.13) by using the fact that

$$
\begin{aligned}
\left\|\chi \partial_{a} \rho_{\varepsilon}\right\|_{L^{2}\left((0, t) \times(0, \infty) \times \mathbb{R}^{2}\right)}^{2} & \leq\|\chi\|_{L^{\infty}(0, \infty)} \int_{0}^{t} \int_{0}^{\infty} \int_{\mathbb{R}^{2}} \chi(a)\left(\partial_{a} \rho_{\varepsilon}\right)^{2} \mathrm{~d} x \mathrm{~d} a \mathrm{~d} s \\
& \leq \int_{0}^{t} \int_{0}^{\infty} \int_{\mathbb{R}^{2}} \chi(a)\left(\partial_{a} \rho_{\varepsilon}\right)^{2} \mathrm{~d} x \mathrm{~d} a \mathrm{~d} s .
\end{aligned}
$$

We consider the class of functions

def :psi

eq:suppxi

q:nonloc1

$\mathrm{q}:$ nonloc2

timate re

eq:re3

$$
\psi_{\varepsilon}(t, x)=\int_{0}^{\infty} \rho_{\varepsilon}(t, a, x) \xi(a) \mathrm{d} a,
$$

for $\xi \in C_{c}^{\infty}((0, \infty))$ such that

$$
\operatorname{supp}(\xi) \subset(0, T) .
$$

In particular any of the functions $\chi_{i}, i=1,2,3$, can play the role of $\xi$, so that the estimates obtained for $\psi_{\varepsilon}$ apply to $\phi_{\varepsilon}$. Thanks to the definition of $\psi_{\varepsilon}$ in (2.15) and the results in (2.12), (2.13), the following inequalities hold for every $t \geq 0$

$$
\begin{array}{r}
\left\|\psi_{\varepsilon}(t, \cdot)\right\|_{L^{1}\left(\mathbb{R}^{2}\right)} \leq e^{C t} C, \\
\left\|\psi_{\varepsilon}(t, \cdot)\right\|_{L^{2}\left(\mathbb{R}^{2}\right)},\left\|\nabla_{x} \psi_{\varepsilon}\right\|_{L^{2}\left((0, t) \times \mathbb{R}^{2}\right)} \leq e^{C t} C .
\end{array}
$$

Lemma $2.5\left(H^{2}\right.$ estimate on $\left.\rho_{\varepsilon}\right)$. For any $t \geq 0$, we have

$$
\left\|\nabla_{x} \rho_{\varepsilon}(t, \cdot, \cdot)\right\|_{L^{2}\left((0, \infty) \times \mathbb{R}^{2}\right)},\left\|D_{x}^{2} \rho_{\varepsilon}\right\|_{L^{2}\left((0, \infty) \times(0, t) \times \mathbb{R}^{2}\right)}, \varepsilon\left\|\chi(a)\left(\nabla_{x} \partial_{a} \rho_{\varepsilon}\right)^{2}\right\|_{L^{1}\left((0, \infty) \times(0, t) \times \mathbb{R}^{2}\right)} \leq e^{C t} C,
$$

Proof. In the proofs, we will use the following remark

$$
Y_{\theta}^{\prime}\left(\phi_{\varepsilon}-R\right) \phi_{\varepsilon}, Y_{\theta}^{\prime \prime}\left(\phi_{\varepsilon}-R\right) \phi_{\varepsilon} \leq C .
$$

We multiply (2.14) by $-\Delta_{x} \rho_{\varepsilon}$ then

$$
\begin{aligned}
& \frac{d}{d t} \int_{0}^{\infty} \int_{\mathbb{R}^{2}} \frac{\left|\nabla_{x} \rho_{\varepsilon}\right|^{2}}{2} \mathrm{~d} x \mathrm{~d} a=\int_{0}^{\infty} \int_{\mathbb{R}^{2}} \nabla_{x} \rho_{\varepsilon} \cdot \partial_{t} \nabla_{x} \rho_{\varepsilon} \mathrm{d} x \mathrm{~d} a=-\int_{0}^{\infty} \int_{\mathbb{R}^{2}} \Delta_{x} \rho_{\varepsilon} \partial_{t} \rho_{\varepsilon} \mathrm{d} x \mathrm{~d} a \\
& =-\mu \int_{0}^{\infty} \int_{\mathbb{R}^{2}}\left|D_{x}^{2} \rho_{\varepsilon}\right|^{2} \mathrm{~d} x \mathrm{~d} a+\int_{0}^{\infty} \int_{\mathbb{R}^{2}} \Delta_{x} \rho_{\varepsilon} \operatorname{div}_{x}\left(\rho_{\varepsilon} \chi_{1} \mathbf{v} Y_{\theta}\right) \mathrm{d} x \mathrm{~d} a+\int_{0}^{\infty} \int_{\mathbb{R}^{2}} \Delta_{x} \rho_{\varepsilon} \partial_{a} \rho_{\varepsilon} \mathrm{d} x \mathrm{~d} a \\
& +\int_{0}^{\infty} \int_{\mathbb{R}^{2}} \rho_{\varepsilon} \underbrace{(-\mathfrak{d}-\mathfrak{p})}_{\leq c} \Delta_{x} \rho_{\varepsilon} \mathrm{d} x \mathrm{~d} a-\varepsilon \int_{0}^{\infty} \int_{\mathbb{R}^{2}} \partial_{a}\left(\chi(a) \partial_{a} \rho_{\varepsilon}\right) \Delta_{x} \rho_{\varepsilon} \mathrm{d} x \mathrm{~d} a \\
& \leq-\frac{\mu}{2} \int_{0}^{\infty} \int_{\mathbb{R}^{2}}\left|D_{x}^{2} \rho_{\varepsilon}\right|^{2} \mathrm{~d} x \mathrm{~d} a+\int_{0}^{\infty} \int_{\mathbb{R}^{2}}\left(\operatorname{div}_{x}\left(\rho_{\varepsilon} \chi_{1} \mathbf{v} Y_{\theta}\right)\right)^{2} \mathrm{~d} x \mathrm{~d} a+\int_{\mathbb{R}^{2}} \frac{\left|\nabla_{x} \rho_{\varepsilon}(t, 0, x)\right|^{2}}{2} \mathrm{~d} x \\
& +c \int_{0}^{\infty} \int_{\mathbb{R}^{2}} \rho_{\varepsilon}^{2} \mathrm{~d} x \mathrm{~d} a-\varepsilon \int_{0}^{\infty} \int_{\mathbb{R}^{2}} \chi(a)\left|\nabla_{x} \partial_{a} \rho_{\varepsilon}\right|^{2} \mathrm{~d} x \mathrm{~d} a \\
& \leq-\frac{\mu}{2} \int_{0}^{\infty} \int_{\mathbb{R}^{2}}\left|D_{x}^{2} \rho_{\varepsilon}\right|^{2} \mathrm{~d} x \mathrm{~d} a-\varepsilon \int_{0}^{\infty} \int_{\mathbb{R}^{2}} \chi(a)\left|\nabla_{x} \partial_{a} \rho_{\varepsilon}\right|^{2} \mathrm{~d} x \mathrm{~d} a+c \int_{0}^{\infty} \int_{\mathbb{R}^{2}} \rho_{\varepsilon}^{2} \mathrm{~d} x \mathrm{~d} a \\
& +c \int_{\mathbb{R}^{2}}\left(\int_{0}^{\infty} \nabla_{x} \rho_{\varepsilon} \cdot \mathbf{v} \chi_{1} Y_{\theta} \mathrm{d} a\right)^{2} \mathrm{~d} x+c \int_{\mathbb{R}^{2}} \underbrace{\left(\int_{0}^{\infty} \rho_{\varepsilon} \chi_{1} \mathrm{~d} a Y_{\theta}^{\prime}\right)^{2}}_{\leq c, \text { since } 2.20}\left(\mathbf{v} \cdot \nabla_{x} \phi_{\varepsilon}\right)^{2} \mathrm{~d} x \\
& +c \int_{\mathbb{R}^{2}}\left(\int_{0}^{\infty} \rho_{\varepsilon} \chi_{1} Y_{\theta} \operatorname{div}_{x}(\mathbf{v}) \mathrm{d} a\right)^{2} \mathrm{~d} x
\end{aligned}
$$




$$
\begin{aligned}
& +c \int_{\mathbb{R}^{2}} \underbrace{\left|\mathcal{A}^{\prime}\left(\phi_{\varepsilon}\right)\right|^{2}\left(\int_{0}^{\infty} \rho_{\varepsilon}(t, a, x) \chi_{3}(a) \mathrm{d} a\right)^{2}}_{\leq c, \text { since } 1.8)}\left|\nabla_{x} \phi_{\varepsilon}\right|^{2} \omega^{2}(t, x) \mathrm{d} x \\
& +c \int_{\mathbb{R}^{2}}\left(\mathcal{A}\left(\phi_{\varepsilon}\right)\left(\int_{0}^{\infty}\left|\nabla_{x} \rho_{\varepsilon}(t, a, x)\right| \chi_{3}(a) \mathrm{d} a\right) \omega(t, x)\right)^{2} \mathrm{~d} x \\
& +c \int_{\mathbb{R}^{2}}\left(\mathcal{A}\left(\phi_{\varepsilon}\right) \int_{0}^{\infty} \rho_{\varepsilon}(t, a, x) \chi_{3}(a) \mathrm{d} a\left|\nabla_{x} \omega(t, x)\right|\right)^{2} \mathrm{~d} x \\
& \leq-\frac{\mu}{2} \int_{0}^{\infty} \int_{\mathbb{R}^{2}}\left|D_{x}^{2} \rho_{\varepsilon}\right|^{2} \mathrm{~d} x \mathrm{~d} a-\varepsilon \int_{0}^{\infty} \int_{\mathbb{R}^{2}} \chi(a)\left|\nabla_{x} \partial_{a} \rho_{\varepsilon}\right|^{2} \mathrm{~d} x \mathrm{~d} a+c \int_{0}^{\infty} \int_{\mathbb{R}^{2}} \rho_{\varepsilon}^{2} \mathrm{~d} x \mathrm{~d} a \\
& +c \int_{0}^{\infty} \int_{\mathbb{R}^{2}}\left|\nabla_{x} \rho_{\varepsilon}\right|^{2} \mathrm{~d} x \mathrm{~d} a+c \int_{\mathbb{R}^{2}}\left|\nabla_{x} \phi_{\varepsilon}\right|^{2} \mathrm{~d} x .
\end{aligned}
$$

Integrating over $(0, t)$, using the Gronwall Lemma, and estimates (2.13), 2.18) we gain 2.19).

Lemma 2.6 ( $L^{\infty}$ estimate on $\left.u_{\varepsilon}\right)$. For every $t \geq 0$, we have

$$
\left\|u_{\varepsilon}(t, \cdot)\right\|_{L^{\infty}\left(\mathbb{R}^{2}\right)} \leq C e^{C t} .
$$

Proof. Let $\mathcal{C}$ be a positive constant that will be fixed later. We define

$$
\bar{u}_{\varepsilon}(t, x)=e^{-\mathcal{C} t} u_{\varepsilon}(t, x),
$$

and we consider the associated problem

$$
\left\{\begin{array}{l}
\partial_{t} \bar{u}_{\varepsilon}+\operatorname{div}_{x}\left(\bar{u}_{\varepsilon} \nu\left(\phi_{\varepsilon}\right)\right)=\left(\mathfrak{b}\left(\phi_{\varepsilon}\right)-\beta-\mathcal{C}\right) \bar{u}_{\varepsilon}+\varepsilon \Delta_{x} \bar{u}_{\varepsilon} \\
\bar{u}_{\varepsilon}(0, x)=u_{0, \varepsilon}(x) .
\end{array}\right.
$$

We claim that for any given $T>0$ there exist a sufficiently large constant $k>0$ and a suitable $\mathcal{C}$ such that $u_{\varepsilon}(t, x) \leq k$ for any $t \leq T$ and $x \in \mathbb{R}^{2}$, provided $u_{0, \varepsilon}(x) \leq k$ for all $x \in \mathbb{R}^{2}$.

Consider the function

$$
x \mapsto \eta(x)=(x-k) \mathbb{1}_{(k, \infty)}(x),
$$

and observe that

$$
\eta^{\prime}(x)=\mathbb{1}_{(k, \infty)}(x), \quad x \eta^{\prime}(x)=\eta(x)+k \eta^{\prime}(x) .
$$

We have

$$
\begin{aligned}
\frac{d}{d t} \int_{\mathbb{R}^{2}} \eta\left(\bar{u}_{\varepsilon}\right) \mathrm{d} x=\int_{\mathbb{R}^{2}} \eta^{\prime}\left(\bar{u}_{\varepsilon}\right) \partial_{t} \bar{u}_{\varepsilon} \mathrm{d} x \\
=-\int_{\mathbb{R}^{2}} \eta^{\prime}\left(\bar{u}_{\varepsilon}\right) \operatorname{div}_{x}\left(\bar{u}_{\varepsilon} \nu\left(\phi_{\varepsilon}\right)\right) \mathrm{d} x+\int_{\mathbb{R}^{2}} \eta^{\prime}\left(\bar{u}_{\varepsilon}\right)\left(\mathfrak{b}\left(\phi_{\varepsilon}\right)-\beta-\mathcal{C}\right) \bar{u}_{\varepsilon} \mathrm{d} x+\underbrace{\varepsilon \int_{\mathbb{R}^{2}} \eta^{\prime}\left(\bar{u}_{\varepsilon}\right) \Delta_{x} \bar{u}_{\varepsilon} \mathrm{d} x}_{\leq 0} \\
\leq-\underbrace{\int_{\mathbb{R}^{2}} \eta^{\prime}\left(\bar{u}_{\varepsilon}\right) \operatorname{div}_{x}\left(\left(\bar{u}_{\varepsilon}-k\right) \nu\left(\phi_{\varepsilon}\right)\right) \mathrm{d} x}_{=0}-k \int_{\mathbb{R}^{2}} \eta^{\prime}\left(\bar{u}_{\varepsilon}\right) \operatorname{div}_{x}\left(\nu\left(\phi_{\varepsilon}\right)\right) \mathrm{d} x \\
\quad+\int_{\mathbb{R}^{2}}\left(\eta\left(\bar{u}_{\varepsilon}\right)+k \eta^{\prime}\left(\bar{u}_{\varepsilon}\right)\right)\left(\mathfrak{b}\left(\phi_{\varepsilon}\right)-\beta-\mathcal{C}\right) \mathrm{d} x \\
\leq \int_{\mathbb{R}^{2}} \eta\left(\bar{u}_{\varepsilon}\right)\left(\mathfrak{b}\left(\phi_{\varepsilon}\right)-\beta-\mathcal{C}\right) \mathrm{d} x-k \int_{\mathbb{R}^{2}} \eta^{\prime}\left(\bar{u}_{\varepsilon}\right)\left(\mathcal{C}+\beta+\operatorname{div}_{x}\left(\nu\left(\phi_{\varepsilon}\right)\right)-\mathfrak{b}\left(\phi_{\varepsilon}\right)\right) \mathrm{d} x
\end{aligned}
$$


From the inequality $\left\|\operatorname{div}_{x}\left(\nu\left(\phi_{\varepsilon}\right)\right)\right\|_{L^{\infty}} \leq 2\left\|\nabla \nu\left(\phi_{\varepsilon}\right)\right\|_{L^{\infty}}$ and the estimates in (2.7) and (2.17), it follows that for $\mathcal{C}$ large enough

$$
\mathcal{C}+\beta-\mathfrak{b}\left(\phi_{\varepsilon}\right) \geq 0, \quad \mathcal{C}+\beta+\operatorname{div}_{x}\left(\nu\left(\phi_{\varepsilon}\right)\right)-\mathfrak{b}\left(\phi_{\varepsilon}\right) \geq 0,
$$

thus,

Integrating over $(0, t)$ we obtain

$$
\frac{d}{d t} \int_{\mathbb{R}^{2}} \eta\left(\bar{u}_{\varepsilon}\right) \mathrm{d} x \leq 0
$$

$$
0 \leq \int_{\mathbb{R}^{2}} \eta\left(\bar{u}_{\varepsilon}(t, x)\right) \mathrm{d} x \leq \int_{\mathbb{R}^{2}} \eta\left(u_{0, \varepsilon}(x)\right) \mathrm{d} x=0,
$$

which means $\bar{u}_{\varepsilon} \leq k$. The inequality (2.21) follows.

ate for $\mathrm{u}$ Lemma 2.7. For all $t \geq 0$, the following estimates on $u_{\varepsilon}$ hold

eq:u1

eq:u2

eq:u3

$$
\begin{array}{r}
\left\|u_{\varepsilon}(t, \cdot)\right\|_{L^{1}\left(\mathbb{R}^{2}\right)} \leq e^{C t} C, \\
\left\|u_{\varepsilon}(t, \cdot)\right\|_{L^{2}\left(\mathbb{R}^{2}\right)},\left\|\nabla_{x} u_{\varepsilon}\right\|_{L^{2}\left((0, t) \times \mathbb{R}^{2}\right)} \leq e^{C t} C, \\
\left\|\nabla_{x} u_{\varepsilon}(t, \cdot)\right\|_{L^{2}\left(\mathbb{R}^{2}\right)},\left\|\Delta_{x} u_{\varepsilon}\right\|_{L^{2}\left((0, t) \times \mathbb{R}^{2}\right)} \leq e^{C t} C .
\end{array}
$$

Proof. We recall the equation of $u_{\varepsilon}$

$$
\partial_{t} u_{\varepsilon}+\operatorname{div}_{x}\left(u_{\varepsilon} \nu\left(\phi_{\varepsilon}\right)\right)=\left(\mathfrak{b}\left(\phi_{\varepsilon}\right)-\beta\right) u_{\varepsilon}+\varepsilon \Delta_{x} u_{\varepsilon} .
$$

(2.22). Using the nonnegativity of $u_{\varepsilon}$ we have

$$
\begin{aligned}
\frac{d}{d t} \int_{\mathbb{R}^{2}}\left|u_{\varepsilon}\right| \mathrm{d} x=\frac{d}{d t} \int_{\mathbb{R}^{2}} u_{\varepsilon} \mathrm{d} x= & \underbrace{\varepsilon \int_{\mathbb{R}^{2}} \Delta_{x} u_{\varepsilon} \mathrm{d} x-\int_{\mathbb{R}^{2}} \operatorname{div} x}_{=0}\left(u_{\varepsilon} \nu\left(\phi_{\varepsilon}\right)\right) \mathrm{d} x \\
& +\int_{\mathbb{R}^{2}} \underbrace{\left(\mathfrak{b}\left(\phi_{\varepsilon}\right)-\beta\right)}_{\leq c} u_{\varepsilon} \mathrm{d} x \leq c \int_{\mathbb{R}^{2}} u_{\varepsilon} \mathrm{d} x .
\end{aligned}
$$

Then, applying the Gronwall Lemma we gain

$$
\left\|u_{\varepsilon}(t, \cdot)\right\|_{L^{1}\left(\mathbb{R}^{2}\right)} \leq\left\|u_{0, \varepsilon}(\cdot)\right\|_{L^{1}\left(\mathbb{R}^{2}\right)} e^{c t} .
$$

(2.23). We multiply 2.25) by $u_{\varepsilon}$ to obtain

$$
\begin{aligned}
& \frac{d}{d t} \int_{\mathbb{R}^{2}} \frac{u_{\varepsilon}^{2}}{2} \mathrm{~d} x=\int_{\mathbb{R}^{2}} u_{\varepsilon} \partial_{t} u_{\varepsilon} \mathrm{d} x \\
& =-\int_{\mathbb{R}^{2}} \operatorname{div}_{x}\left(u_{\varepsilon} \nu\left(\phi_{\varepsilon}\right)\right) u_{\varepsilon} \mathrm{d} x+\int_{\mathbb{R}^{2}}\left(\mathfrak{b}\left(\phi_{\varepsilon}\right)-\beta\right) u_{\varepsilon}^{2} \mathrm{~d} x+\varepsilon \int_{\mathbb{R}^{2}} \Delta_{x} u_{\varepsilon} u_{\varepsilon} \mathrm{d} x \\
& \leq \int_{\mathbb{R}^{2}} u_{\varepsilon} \nu\left(\phi_{\varepsilon}\right) \cdot \nabla u_{\varepsilon} \mathrm{d} x+c \int_{\mathbb{R}^{2}} u_{\varepsilon}^{2} \mathrm{~d} x-\varepsilon \int_{\mathbb{R}^{2}}\left|\nabla_{x} u_{\varepsilon}\right|^{2} \mathrm{~d} x \\
& \leq \int_{\mathbb{R}^{2}} \nabla_{x}\left(\frac{u_{\varepsilon}^{2}}{2}\right) \cdot \nu\left(\phi_{\varepsilon}\right) \mathrm{d} x+c \int_{\mathbb{R}^{2}} u_{\varepsilon}^{2} \mathrm{~d} x-\varepsilon \int_{\mathbb{R}^{2}}\left|\nabla_{x} u_{\varepsilon}\right|^{2} \mathrm{~d} x \\
& \leq \int_{\mathbb{R}^{2}} \frac{u_{\varepsilon}^{2}}{2} \operatorname{div}_{x}\left(\nu\left(\phi_{\varepsilon}\right)\right) \mathrm{d} x+c \int_{\mathbb{R}^{2}} u_{\varepsilon}^{2} \mathrm{~d} x-\varepsilon \int_{\mathbb{R}^{2}}\left|\nabla_{x} u_{\varepsilon}\right|^{2} \mathrm{~d} x \\
& \leq c_{T} \int_{\mathbb{R}^{2}}\left|u_{\varepsilon}\right| \mathrm{d} x+c \int_{\mathbb{R}^{2}} u_{\varepsilon}^{2} \mathrm{~d} x-\varepsilon \int_{\mathbb{R}^{2}}\left|\nabla_{x} u_{\varepsilon}\right|^{2} \mathrm{~d} x .
\end{aligned}
$$

Integrating over $(0, t)$ and then using the Gronwall Lemma we get

$$
\left\|u_{\varepsilon}(t, \cdot)\right\|_{L^{2}\left(\mathbb{R}^{2}\right)}^{2}+2 \varepsilon e^{c t} \int_{0}^{t} e^{-c s}\left\|\nabla_{x} u_{\varepsilon}(s, \cdot)\right\|_{L^{2}\left(\mathbb{R}^{2}\right)}^{2} \mathrm{~d} s \leq e^{c t}\left\|u_{\varepsilon}(0, \cdot)\right\|_{L^{2}\left(\mathbb{R}^{2}\right)}^{2}+c e^{c t} .
$$


(2.24). We multiply 2.25$)$ by $-\Delta_{x} u_{\varepsilon}$ to obtain

$$
\begin{aligned}
\frac{d}{d t} \int_{\mathbb{R}^{2}} \frac{\left|\nabla_{x} u_{\varepsilon}\right|^{2}}{2} \mathrm{~d} x=\int_{\mathbb{R}^{2}} \nabla_{x} u_{\varepsilon} \cdot \partial_{t} \nabla_{x} u_{\varepsilon} \mathrm{d} x=-\int_{\mathbb{R}^{2}} \Delta_{x} u_{\varepsilon} \partial_{t} u_{\varepsilon} \mathrm{d} x \\
=\int_{\mathbb{R}^{2}} \Delta_{x} u_{\varepsilon} \operatorname{div}_{x}\left(u_{\varepsilon} \nu\left(\phi_{\varepsilon}\right)\right) \mathrm{d} x-\int_{\mathbb{R}^{2}} \Delta_{x} u_{\varepsilon}\left(\mathfrak{b}\left(\phi_{\varepsilon}\right)-\beta\right) u_{\varepsilon} \mathrm{d} x-\varepsilon \int_{\mathbb{R}^{2}}\left|\Delta_{x} u_{\varepsilon}\right|^{2} \mathrm{~d} x \\
=-\int_{\mathbb{R}^{2}} \nabla_{x} u_{\varepsilon} \nabla_{x}\left(\nabla_{x} u_{\varepsilon} \cdot \nu(\phi)+u_{\varepsilon} \operatorname{div}_{x}(\nu(\phi))\right) \mathrm{d} x+\int_{\mathbb{R}^{2}}(\mathfrak{b}(\phi)-\beta)\left|\nabla_{x} u_{\varepsilon}\right|^{2} \mathrm{~d} x \\
\quad+\int_{\mathbb{R}^{2}} \mathfrak{b}^{\prime}(\phi) \nabla_{x} \phi \cdot \nabla_{x} u_{\varepsilon} u_{\varepsilon} \mathrm{d} x-\varepsilon \int_{\mathbb{R}^{2}}\left|\Delta_{x} u_{\varepsilon}\right|^{2} \mathrm{~d} x \\
\leq-\varepsilon \int_{\mathbb{R}^{2}}\left|\Delta_{x} u_{\varepsilon}\right|^{2} \mathrm{~d} x+c \int_{\mathbb{R}^{2}}\left|\nabla_{x} u_{\varepsilon}\right|^{2} d x+c_{T} \int_{\mathbb{R}^{2}}\left|\nabla_{x} \psi_{\varepsilon}\right|^{2} d x \\
\quad+\int_{\mathbb{R}^{2}} \nabla_{x}\left(\frac{\left|\nabla_{x} u_{\varepsilon}\right|^{2}}{2}\right) \cdot \nu(\phi) \mathrm{d} x+\int_{\mathbb{R}^{2}}\left|\nabla_{x} u\right|^{2} \underbrace{\left|\nabla_{x} \nu(\phi)\right|}_{\leq c_{T}} \mathrm{~d} x+\int_{\mathbb{R}^{2}}\left|\nabla_{x} u\right|^{2} \underbrace{\left|\operatorname{div}_{x}(\nu(\phi))\right|}_{\leq c_{T}} \mathrm{~d} x \\
\quad+\int_{\mathbb{R}^{2}}\left|\nabla_{x} u_{\varepsilon}\right| u_{\varepsilon}\left|\nabla_{x} \operatorname{div} x(\nu(\phi))\right| \mathrm{d} x \\
\leq-\varepsilon \int_{\mathbb{R}^{2}}\left|\Delta_{x} u_{\varepsilon}\right|^{2} \mathrm{~d} x+c_{T} \int_{\mathbb{R}^{2}}\left|\nabla_{x} u_{\varepsilon}\right|^{2} \mathrm{~d} x+c_{T} \int_{\mathbb{R}^{2}}\left|\nabla_{x} \psi_{\varepsilon}\right|^{2} \mathrm{~d} x+c_{T} \int_{\mathbb{R}^{2}}\left|\nabla_{x} \operatorname{div} x(\nu(\phi))\right|^{2} \mathrm{~d} x .
\end{aligned}
$$

Integrating over $(0, t)$ and using the estimates in 2.9 and 2.18 we gain

$$
\left\|\nabla_{x} u_{\varepsilon}(t, \cdot)\right\|_{L^{2}\left(\mathbb{R}^{2}\right)}+\varepsilon\left\|\Delta_{x} u_{\varepsilon}(\cdot, \cdot)\right\|_{L^{2}\left((0, t) \times \mathbb{R}^{2}\right)} \leq c e^{c t} .
$$

r phi_e 3 Lemma 2.8. For every $t \geq 0$, the following estimates on $\psi_{\varepsilon}$ hold

nonloc2.1

nonloc2.2

nonloc2.3

nonloc2. 4

nonloc2.5

eq:psi

$$
\begin{aligned}
\left\|\nabla_{x} \psi_{\varepsilon}(t, \cdot)\right\|_{L^{2}\left(\mathbb{R}^{2}\right)},\left\|D_{x}^{2} \psi_{\varepsilon}\right\|_{L^{2}\left((0, t) \times \mathbb{R}^{2}\right)} & \leq e^{C t} C, \\
\left\|D_{x}^{2} \psi_{\varepsilon}(t, \cdot)\right\|_{L^{2}\left(\mathbb{R}^{2}\right)},\left\|D_{x}^{3} \psi_{\varepsilon}\right\|_{L^{2}\left((0, t) \times \mathbb{R}^{2}\right)} & \leq e^{C t} C, \\
\left\|\psi_{\varepsilon}(t, \cdot)\right\|_{L^{\infty}\left(\mathbb{R}^{2}\right)} & \leq e^{C t} C, \\
\left\|D_{x}^{3} \psi_{\varepsilon}(t, \cdot)\right\|_{L^{2}\left(\mathbb{R}^{2}\right)},\left\|D_{x}^{4} \psi_{\varepsilon}\right\|_{L^{2}\left((0, t) \times \mathbb{R}^{2}\right)} \leq e^{C t} C, & \leq{ }^{C t} C .
\end{aligned}
$$

Proof. We multiply by $\xi(a)$ the equation for $\rho_{\varepsilon}$ in system (2.1) then, integrating with respect to $a$, we get

$$
\begin{aligned}
& \partial_{t} \psi_{\varepsilon}-\mu \Delta_{x} \psi_{\varepsilon}+\operatorname{div}_{x}\left(\left(\int_{0}^{\infty} \rho_{\varepsilon} \chi_{1} \xi \mathrm{d} a\right) \mathbf{v} Y_{\theta}\right) \\
& \quad=-\int_{0}^{\infty}\left(\mathfrak{d}+\mathfrak{p}\left(a, u_{\varepsilon}\right)\right) \rho_{\varepsilon} \xi \mathrm{d} a+\int_{0}^{\infty} \rho_{\varepsilon}\left(\left(1+\varepsilon \chi^{\prime}\right) \xi^{\prime}+\varepsilon \chi \xi^{\prime \prime}\right) \mathrm{d} a .
\end{aligned}
$$

(2.26). We multiply 2.31 by $-\Delta_{x} \psi_{\varepsilon}$

$$
\begin{aligned}
& \frac{d}{d t} \int_{\mathbb{R}^{2}} \frac{\left|\nabla_{x} \psi_{\varepsilon}\right|^{2}}{2} \mathrm{~d} x=\int_{\mathbb{R}^{2}} \nabla_{x} \psi_{\varepsilon} \cdot \partial_{t} \nabla_{x} \psi_{\varepsilon} \mathrm{d} x=-\int_{\mathbb{R}^{2}} \Delta_{x} \psi_{\varepsilon} \partial_{t} \psi_{\varepsilon} \mathrm{d} x \\
& =-\mu \int_{\mathbb{R}^{2}}\left|D_{x}^{2} \psi_{\varepsilon}\right|^{2} \mathrm{~d} x+\int_{\mathbb{R}^{2}} \Delta_{x} \psi_{\varepsilon} \operatorname{div}_{x}\left(\left(\int_{0}^{\infty} \rho_{\varepsilon} \chi_{1} \xi \mathrm{d} a\right) \mathbf{v} Y_{\theta}\right) \mathrm{d} x+\int_{\mathbb{R}^{2}} \int_{0}^{\infty} \rho_{\varepsilon} \xi \underbrace{(\mathfrak{d}+\mathfrak{p})}_{\leq c} \Delta_{x} \psi_{\varepsilon} \mathrm{d} a \mathrm{~d} x \\
& \quad-\int_{\mathbb{R}^{2}} \int_{0}^{\infty} \rho_{\varepsilon} \underbrace{\left(\left(1+\varepsilon \chi^{\prime}\right) \xi^{\prime}+\varepsilon \chi \xi^{\prime \prime}\right)}_{\leq c} \Delta_{x} \psi_{\varepsilon} \mathrm{d} a \mathrm{~d} x \\
& \leq-\frac{\mu}{2} \int_{\mathbb{R}^{2}}\left|D_{x}^{2} \psi_{\varepsilon}\right|^{2} \mathrm{~d} x+c \int_{\mathbb{R}^{2}}\left(\operatorname{div}_{x}\left(\left(\int_{0}^{\infty} \rho_{\varepsilon} \chi_{1} \xi \mathrm{d} a\right) \mathbf{v} Y_{\theta}\right)\right)^{2} \mathrm{~d} x+c \int_{\mathbb{R}^{2}} \psi_{\varepsilon}^{2} \mathrm{~d} x+c \int_{0}^{\infty} \int_{\mathbb{R}^{2}} \rho_{\varepsilon}^{2} \mathrm{~d} x \mathrm{~d} a
\end{aligned}
$$


12

G. M. COClite, C. DONADELlo, AND T. N. T. NGUYEN

$$
\begin{aligned}
\leq & -\frac{\mu}{2} \int_{\mathbb{R}^{2}}\left|D_{x}^{2} \psi_{\varepsilon}\right|^{2} \mathrm{~d} x+c \int_{\mathbb{R}^{2}}\left|\nabla_{x} \int_{0}^{\infty} \rho_{\varepsilon} \chi_{1} \xi \mathrm{d} a\right|^{2} \underbrace{|\mathbf{v}|^{2} Y_{\theta}^{2}}_{\leq c} \mathrm{~d} x \\
& +c \int_{\mathbb{R}^{2}}\left(\int_{0}^{\infty} \rho_{\varepsilon} \chi_{1} \xi \mathrm{d} a\right)^{2} \underbrace{\left|\operatorname{div}_{x}(\mathbf{v})\right|^{2} Y_{\theta}^{2}}_{\leq c} \mathrm{~d} x+c \int_{\mathbb{R}^{2}} \underbrace{\left(\int_{0}^{\infty} \rho_{\varepsilon} \chi_{1} \xi \mathrm{d} a\right)^{2}\left(Y_{\theta}^{\prime}\right)^{2}|\mathbf{v}|^{2}}_{\leq c(\text { see } \sqrt{2.16})}\left|\nabla_{x} \phi_{\varepsilon}\right|^{2} \mathrm{~d} x \\
& +c \int_{\mathbb{R}^{2}} \psi_{\varepsilon}^{2} \mathrm{~d} x+c \int_{0}^{\infty} \int_{\mathbb{R}^{2}} \rho_{\varepsilon}^{2} \mathrm{~d} x \mathrm{~d} a .
\end{aligned}
$$

Since the functions

$$
\phi_{\varepsilon}, \quad(t, x) \mapsto \int_{0}^{\infty} \rho_{\varepsilon} \chi_{1} \xi \mathrm{d} a
$$

have the same structure as $\psi_{\varepsilon}$, using the Gronwall Lemma and estimates (2.13), (2.18) we get (2.26).

(2.27). We multiply 2.31 by $D_{x}^{4} \psi_{\varepsilon}$ to have

$$
\begin{aligned}
& \frac{d}{d t} \int_{\mathbb{R}^{2}} \frac{\left|\Delta_{x} \psi_{\varepsilon}\right|^{2}}{2} \mathrm{~d} x=\int_{\mathbb{R}^{2}} \Delta_{x} \psi_{\varepsilon} \cdot \partial_{t} \Delta_{x} \psi_{\varepsilon} \mathrm{d} x=\int_{\mathbb{R}^{2}} D_{x}^{4} \psi_{\varepsilon} \partial_{t} \psi_{\varepsilon} \mathrm{d} x \\
& =\mu \int_{\mathbb{R}^{2}} \Delta_{x} \psi_{\varepsilon} D_{x}^{4} \psi_{\varepsilon} \mathrm{d} x-\int_{\mathbb{R}^{2}} D_{x}^{4} \psi_{\varepsilon} \operatorname{div}_{x}\left(\left(\int_{0}^{\infty} \rho_{\varepsilon} \chi_{1} \xi \mathrm{d} a\right) \mathbf{v} Y_{\theta}\right) \mathrm{d} x \\
& +\int_{\mathbb{R}^{2}} \int_{0}^{\infty} \rho_{\varepsilon} \xi\left(-\mathfrak{d}-\mathfrak{p}\left(a, u_{\varepsilon}\right)\right) D_{x}^{4} \psi_{\varepsilon} \mathrm{d} a \mathrm{~d} x+\int_{\mathbb{R}^{2}} \int_{0}^{\infty} \rho_{\varepsilon}\left(\left(1+\varepsilon \chi^{\prime}\right) \xi^{\prime}+\varepsilon \chi \xi^{\prime \prime}\right) D_{x}^{4} \psi_{\varepsilon} \mathrm{d} a \mathrm{~d} x \\
& =-\mu \int_{\mathbb{R}^{2}}\left|D_{x}^{3} \psi_{\varepsilon}\right|^{2} \mathrm{~d} x+\int_{\mathbb{R}^{2}} D_{x}^{3} \psi_{\varepsilon} \cdot \nabla_{x} \operatorname{div}_{x}\left(\left(\int_{0}^{\infty} \rho_{\varepsilon} \chi_{1} \xi \mathrm{d} a\right) \mathbf{v} Y_{\theta}\right) \mathrm{d} x \\
& -\int_{\mathbb{R}^{2}} \nabla_{x}\left(\int_{0}^{\infty} \rho_{\varepsilon} \xi\left(-\mathfrak{d}-\mathfrak{p}\left(a, u_{\varepsilon}\right)\right) \mathrm{d} a\right) \cdot D_{x}^{3} \psi_{\varepsilon} \mathrm{d} x+\int_{\mathbb{R}^{2}} \int_{0}^{\infty} \nabla_{x} \rho_{\varepsilon} \underbrace{\left(\left(1+\varepsilon \chi^{\prime}\right) \xi^{\prime}+\varepsilon \chi \xi^{\prime \prime}\right)}_{\leq c} \cdot D_{x}^{3} \psi_{\varepsilon} \mathrm{d} a \mathrm{~d} x \\
& \leq-\frac{\mu}{2} \int_{\mathbb{R}^{2}}\left|D_{x}^{3} \psi_{\varepsilon}\right|^{2} \mathrm{~d} x+c \int_{\mathbb{R}^{2}}\left(\nabla_{x} \operatorname{div}_{x}\left(\left(\int_{0}^{\infty} \rho_{\varepsilon} \chi_{1} \xi \mathrm{d} a\right) \mathbf{v} Y_{\theta}\right)\right)^{2} \mathrm{~d} x \\
& +c \int_{\mathbb{R}^{2}}\left|\nabla_{x} \int_{0}^{\infty} \rho_{\varepsilon} \xi\left(-\mathfrak{d}-\mathfrak{p}\left(a, u_{\varepsilon}\right)\right) \mathrm{d} a\right|^{2} \mathrm{~d} x+c \int_{0}^{\infty} \int_{\mathbb{R}^{2}}\left|\nabla_{x} \rho_{\varepsilon}\right|^{2} \mathrm{~d} x \mathrm{~d} a \\
& \leq-\frac{\mu}{2} \int_{\mathbb{R}^{2}}\left|D_{x}^{3} \psi_{\varepsilon}\right|^{2} \mathrm{~d} x+c \int_{\mathbb{R}^{2}}\left|D_{x}^{2} \int_{0}^{\infty} \rho_{\varepsilon} \chi_{1} \xi \mathrm{d} a\right|^{2} \underbrace{|\mathbf{v}|^{2} Y_{\theta}^{2}}_{\leq c} \mathrm{~d} x+c \int_{\mathbb{R}^{2}}\left|\int_{0}^{\infty} \rho_{\varepsilon} \chi_{1} \xi \mathrm{d} a\right|^{2} \underbrace{\left|D_{x}^{2} \mathbf{v}\right|^{2} Y_{\theta}^{2}}_{\leq c} \mathrm{~d} x \\
& +c \int_{\mathbb{R}^{2}} \underbrace{\left|\int_{0}^{\infty} \rho_{\varepsilon} \chi_{1} \xi \mathrm{d} a\right|^{2}|\mathbf{v}|^{2}\left(Y_{\theta}^{\prime \prime}\right)^{2}}_{\leq c, \text { see }}\left|\nabla_{x} \phi_{\varepsilon}\right|^{4} \mathrm{~d} x+c \int_{\mathbb{R}^{2}} \underbrace{\left|\int_{0}^{\infty} \rho_{\varepsilon} \chi_{1} \xi \mathrm{d} a\right|^{2}|\mathbf{v}|^{2}\left(Y_{\theta}^{\prime}\right)^{2}}_{\leq c, \text { see } 2.16}\left|D_{x}^{2} \phi_{\varepsilon}\right|^{2} \mathrm{~d} x \\
& +c \int_{\mathbb{R}^{2}}\left|\nabla_{x} \int_{0}^{\infty} \rho_{\varepsilon} \chi_{1} \xi \mathrm{d} a\right|^{2} \underbrace{\left|\nabla_{x} \mathbf{v}\right|^{2} Y_{\theta}^{2}}_{\leq c} \mathrm{~d} x+\left.c \int_{\mathbb{R}^{2}} \underbrace{\mid \int_{0}^{\infty .16}}_{\leq c, \text { see }} \rho_{\varepsilon} \chi_{1} \xi \mathrm{d} a\right|^{2}\left|\nabla_{x} \mathbf{v}\right|^{2}\left(Y_{\theta}^{\prime}\right)^{2}\left|\nabla_{x} \phi_{\varepsilon}\right|^{2} \mathrm{~d} x \\
& +c \int_{\mathbb{R}^{2}}\left|\nabla_{x} \int_{0}^{\infty} \rho_{\varepsilon} \chi_{1} \xi \mathrm{d} a\right|^{2} \underbrace{|\mathbf{v}|^{2}\left(Y_{\theta}^{\prime}\right)^{2}}_{\leq c}\left|\nabla_{x} \phi_{\varepsilon}\right|^{2} \mathrm{~d} x+c \int_{\mathbb{R}^{2}}(\int_{0}^{\infty} \rho_{\varepsilon} \xi \underbrace{\left|\nabla_{x} \mathfrak{d}\right|}_{\leq c} \mathrm{~d} a)^{2} d x \\
& +c \int_{\mathbb{R}^{2}}|\int_{0}^{\infty} \nabla_{x} \rho_{\varepsilon} \xi \underbrace{\left(\mathfrak{d}+\mathfrak{p}\left(a, u_{\varepsilon}\right)\right)}_{\leq c} \mathrm{~d} a|^{2} \mathrm{~d} x+c \int_{\mathbb{R}^{2}}|\int_{0}^{\infty} \rho_{\varepsilon} \underbrace{\partial_{u} \mathfrak{p}\left(a, u_{\varepsilon}\right)}_{\leq c} \nabla_{x} u_{\varepsilon} \xi \mathrm{d} a|^{2} \mathrm{~d} x \\
& +c \int_{0}^{\infty} \int_{\mathbb{R}^{2}}\left|\nabla_{x} \rho_{\varepsilon}\right|^{2} \mathrm{~d} x \mathrm{~d} a
\end{aligned}
$$




$$
\begin{aligned}
\leq & -\frac{\mu}{2} \int_{\mathbb{R}^{2}}\left|D_{x}^{3} \psi_{\varepsilon}\right|^{2} \mathrm{~d} x+c \int_{\mathbb{R}^{2}}\left|D_{x}^{2} \int_{0}^{\infty} \rho_{\varepsilon} \chi_{1} \xi \mathrm{d} a\right|^{2} \mathrm{~d} x+c \int_{\mathbb{R}^{2}}\left|\int_{0}^{\infty} \rho_{\varepsilon} \chi_{1} \xi \mathrm{d} a\right|^{2} \mathrm{~d} x \\
& +c \int_{\mathbb{R}^{2}}\left|\nabla_{x} \int_{0}^{\infty} \rho_{\varepsilon} \chi_{1} \xi \mathrm{d} a\right|^{2} \mathrm{~d} x+c \int_{\mathbb{R}^{2}}\left|\nabla_{x} \phi_{\varepsilon}\right|^{2} \mathrm{~d} x+c \int_{\mathbb{R}^{2}}\left|\nabla_{x} \phi_{\varepsilon}\right|^{4} \mathrm{~d} x \\
& +c \int_{\mathbb{R}^{2}}\left|D_{x}^{2} \phi_{\varepsilon}\right|^{2} \mathrm{~d} x+c \int_{\mathbb{R}^{2}}\left|\nabla_{x} \int_{0}^{\infty} \rho_{\varepsilon} \chi_{1} \xi \mathrm{d} a\right|^{4} \mathrm{~d} x+c \int_{\mathbb{R}^{2}} \psi_{\varepsilon}^{2} \mathrm{~d} x+ \\
& +c \int_{\mathbb{R}^{2}}\left|\nabla_{x} \psi_{\varepsilon}\right|^{2} \mathrm{~d} x+c \int_{\mathbb{R}^{2}} \psi_{\varepsilon}^{4} \mathrm{~d} x+c \int_{\mathbb{R}^{2}}\left|\nabla_{x} u_{\varepsilon}\right|^{4} \mathrm{~d} x+c \int_{0}^{\infty} \int_{\mathbb{R}^{2}}\left|\nabla_{x} \rho_{\varepsilon}\right|^{2} \mathrm{~d} x \mathrm{~d} a
\end{aligned}
$$

Integrating over $(0, t)$ we get

$$
\begin{aligned}
& \left\|D_{x}^{2} \psi_{\varepsilon}(t, \cdot)\right\|_{L^{2}\left(\mathbb{R}^{2}\right)}^{2}+\mu\left\|D_{x}^{3} \psi_{\varepsilon}\right\|_{L^{2}\left((0, t) \times \mathbb{R}^{2}\right)}^{2} \\
& \leq\left\|D_{x}^{2} \psi_{\varepsilon}(0, \cdot)\right\|_{L^{2}\left(\mathbb{R}^{2}\right)}^{2}+c \int_{0}^{t}\left\|\left(\int_{0}^{\infty} \rho_{\varepsilon} \chi_{1} \xi d a\right)(s)\right\|_{H^{2}\left(\mathbb{R}^{2}\right)}^{2} \mathrm{~d} s+c \int_{0}^{t}\left\|\phi_{\varepsilon}(s, \cdot)\right\|_{H^{2}\left(\mathbb{R}^{2}\right)}^{2} \mathrm{~d} s \\
& \quad+c \int_{0}^{t}\left\|\left(\nabla_{x} \int_{0}^{\infty} \rho_{\varepsilon} \chi_{1} \xi \mathrm{d} a\right)(s)\right\|_{L^{4}\left(\mathbb{R}^{2}\right)}^{4} \mathrm{~d} s+c \int_{0}^{t}\left\|\nabla_{x} \phi_{\varepsilon}(s, \cdot)\right\|_{L^{4}\left(\mathbb{R}^{2}\right)}^{4} \mathrm{~d} s \\
& \quad+c \int_{0}^{t}\left\|\psi_{\varepsilon}(s, \cdot)\right\|_{L^{2}\left(\mathbb{R}^{2}\right)}^{2} \mathrm{~d} s+c \int_{0}^{t}\left\|\nabla_{x} \rho_{\varepsilon}(s, \cdot, \cdot)\right\|_{L^{2}\left((0, \infty) \times \mathbb{R}^{2}\right)}^{2} \mathrm{~d} s \\
& \quad+c \int_{0}^{t}\left\|\nabla_{x} \psi_{\varepsilon}(s, \cdot)\right\|_{L^{2}\left(\mathbb{R}^{2}\right)}^{2} \mathrm{~d} s+c \int_{0}^{t} \int_{\mathbb{R}^{2}}\left\|\psi_{\varepsilon}(s, \cdot)\right\|_{L^{4}\left(\mathbb{R}^{2}\right)}^{4} d s \\
& \quad+c \int_{0}^{t}\left\|\nabla_{x} u_{\varepsilon}(s, \cdot)\right\|_{L^{4}\left(\mathbb{R}^{2}\right)}^{4} \mathrm{~d} s+c \int_{0}^{t}\left\|\nabla_{x} \rho_{\varepsilon}(s, \cdot, \cdot)\right\|_{L^{2}\left((0, \infty) \times \mathbb{R}^{2}\right)}^{2} \mathrm{~d} s .
\end{aligned}
$$

Using (2.13), 2.18), 2.26), 2.24) and the embedding $H^{1}\left(\mathbb{R}^{2}\right) \subset L^{4}\left(\mathbb{R}^{2}\right)$ we gain (2.27).

(2.28). Directly follows from 2.18, 2.26), 2.27) and the embedding $H^{2}\left(\mathbb{R}^{2}\right) \subset L^{\infty}\left(\mathbb{R}^{2}\right)$.

(2.29). We multiply 2.31) by $-D_{x}^{6} \psi_{\varepsilon}$

$$
\begin{aligned}
\frac{d}{d t} & \int_{\mathbb{R}^{2}} \frac{\left|D_{x}^{3} \psi_{\varepsilon}\right|^{2}}{2} \mathrm{~d} x=\int_{\mathbb{R}^{2}} D_{x}^{3} \psi_{\varepsilon} \cdot \partial_{t} D_{x}^{3} \psi_{\varepsilon} \mathrm{d} x=-\int_{\mathbb{R}^{2}} D_{x}^{6} \psi_{\varepsilon} \partial_{t} \psi_{\varepsilon} \mathrm{d} x \\
= & \left.-\mu \int_{\mathbb{R}^{2}} \Delta_{x} \psi_{\varepsilon} D_{x}^{6} \psi_{\varepsilon} \mathrm{d} x+\int_{\mathbb{R}^{2}} D_{x}^{6} \psi_{\varepsilon} \operatorname{div}{ }_{x}\left(\int_{0}^{\infty} \rho_{\varepsilon} \chi_{1} \xi \mathrm{d} a\right) \mathbf{v} Y_{\theta}\right) \mathrm{d} x \\
& -\int_{\mathbb{R}^{2}} \int_{0}^{\infty} \rho_{\varepsilon} \xi\left(-\mathfrak{d}-\mathfrak{p}\left(a, u_{\varepsilon}\right)\right) D_{x}^{6} \psi_{\varepsilon} \mathrm{d} a \mathrm{~d} x-\int_{\mathbb{R}^{2}} \int_{0}^{\infty} \rho_{\varepsilon} \underbrace{\left(\left(1+\varepsilon \chi^{\prime}\right) \xi^{\prime}+\varepsilon \chi \xi^{\prime \prime}\right)}_{\leq c} D_{x}^{6} \psi_{\varepsilon} \mathrm{d} a \mathrm{~d} x \\
= & -\mu \int_{\mathbb{R}^{2}}\left|D_{x}^{4} \psi_{\varepsilon}\right|^{2} \mathrm{~d} x+\int_{\mathbb{R}^{2}} D_{x}^{4} \psi_{\varepsilon} \Delta_{x} \operatorname{div} x\left(\left(\int_{0}^{\infty} \rho_{\varepsilon} \chi_{1} \xi \mathrm{d} a\right) \mathbf{v} Y_{\theta}\right) \mathrm{d} x \\
& -\int_{\mathbb{R}^{2}} \Delta_{x}\left(\int_{0}^{\infty} \rho_{\varepsilon} \xi\left(-\mathfrak{d}-\mathfrak{p}\left(a, u_{\varepsilon}\right)\right) \mathrm{d} a\right) D_{x}^{4} \psi_{\varepsilon} \mathrm{d} x \\
& -\int_{\mathbb{R}^{2}} \Delta_{x}\left(\int_{0}^{\infty} \rho_{\varepsilon}\left(\left(1+\varepsilon \chi^{\prime}\right) \xi^{\prime}+\varepsilon \chi \xi^{\prime \prime}\right) \mathrm{d} a\right) D_{x}^{4} \psi_{\varepsilon} \mathrm{d} x \\
\leq & -\frac{\mu}{2} \int_{\mathbb{R}^{2}}\left|D_{x}^{4} \psi_{\varepsilon}\right|^{2} \mathrm{~d} x+c \int_{\mathbb{R}^{2}}\left(\Delta_{x} \operatorname{div}{ }_{x}\left(\left(\int_{0}^{\infty} \rho_{\varepsilon} \chi_{1} \xi \mathrm{d} a\right) \mathbf{v} Y_{\theta}\right)\right)^{2} \mathrm{~d} x \\
& +c \int_{\mathbb{R}^{2}}\left|\Delta_{x}\left(\int_{0}^{\infty} \rho_{\varepsilon} \mathrm{d} \xi \mathrm{d} a\right)\right|^{2} \mathrm{~d} x+c \int_{\mathbb{R}^{2}}\left|\Delta_{x}\left(\int_{0}^{\infty} \rho_{\varepsilon} \mathfrak{p}\left(a, u_{\varepsilon}\right) \xi \mathrm{d} a\right)\right|^{2} \mathrm{~d} x+c \int_{\mathbb{R}^{2}} \int_{0}^{\infty}\left|\Delta_{x} \rho_{\varepsilon}\right|^{2} \mathrm{~d} a \mathrm{~d} x \\
\leq & -\frac{\mu}{2} \int_{\mathbb{R}^{2}}\left|D_{x}^{4} \psi_{\varepsilon}\right|^{2} \mathrm{~d} x+c \int_{\mathbb{R}^{2}}\left|D_{x}^{3} \int_{0}^{\infty} \rho_{\varepsilon} \chi_{1} \xi \mathrm{d} a\right|^{2} \underbrace{|\mathbf{v}|^{2} Y_{\theta}^{2}}_{\leq c} \mathrm{~d} x
\end{aligned}
$$




$$
\begin{aligned}
& +c \int_{\mathbb{R}^{2}}\left|D_{x}^{2} \int_{0}^{\infty} \rho_{\varepsilon} \chi_{1} \xi \mathrm{d} a\right|^{2} \underbrace{\left|\nabla_{x} \mathbf{v}\right|^{2} Y_{\theta}^{2}}_{\leq c} \mathrm{~d} x+c \int_{\mathbb{R}^{2}}\left|\nabla_{x} \int_{0}^{\infty} \rho_{\varepsilon} \chi_{1} \xi \mathrm{d} a\right|^{2} \underbrace{\left|D_{x}^{2} \mathbf{v}\right|^{2} Y_{\theta}^{2}}_{\leq c} \mathrm{~d} x \\
& +c \int_{\mathbb{R}^{2}}\left|\int_{0}^{\infty} \rho_{\varepsilon} \chi_{1} \xi \mathrm{d} a\right|^{2} \underbrace{\left|D_{x}^{3} \mathbf{v}\right|^{2} Y_{\theta}^{2}}_{\leq c} \mathrm{~d} x+c \int_{\mathbb{R}^{2}}\left|D_{x}^{2} \int_{0}^{\infty} \rho_{\varepsilon} \chi_{1} \xi \mathrm{d} a\right|^{2} \underbrace{|\mathbf{v}|^{2}\left(Y_{\theta}^{\prime}\right)^{2}}_{\leq c}\left|\nabla_{x} \phi_{\varepsilon}\right|^{2} \mathrm{~d} x \\
& +c \int_{\mathbb{R}^{2}} \underbrace{\left|\int_{0}^{\infty} \rho_{\varepsilon} \chi \chi_{1} \xi \mathrm{d} a\right|^{2}\left|D_{x}^{2} \mathbf{v}\right|^{2}\left(Y_{\theta}^{\prime}\right)^{2}}_{\leq c}\left|\nabla_{x} \phi_{\varepsilon}\right|^{2} \mathrm{~d} x+c \int_{\mathbb{R}^{2}}\left|\nabla_{x} \int_{0}^{\infty} \rho_{\varepsilon} \chi_{1} \xi \mathrm{d} a\right|^{2} \underbrace{\left|\nabla_{x} \mathbf{v}\right|^{2}\left(Y_{\theta}^{\prime}\right)^{2}}_{\leq c}\left|\nabla_{x} \phi_{\varepsilon}\right|^{2} \mathrm{~d} x \\
& +c \int_{\mathbb{R}^{2}}\left|\nabla_{x} \int_{0}^{\infty} \rho_{\varepsilon} \chi_{1} \xi \mathrm{d} a\right|^{2} \underbrace{|\mathbf{v}|^{2}\left(Y_{\theta}^{\prime \prime}\right)^{2}}_{\leq c}\left|\nabla_{x} \phi_{\varepsilon}\right|^{4} \mathrm{~d} x+c \int_{\mathbb{R}^{2}}\left|\nabla_{x} \int_{0}^{\infty} \rho_{\varepsilon} \chi_{1} \xi \mathrm{d} a\right|^{2} \underbrace{|\mathbf{v}|^{2}\left(Y_{\theta}^{\prime}\right)^{2}}_{\leq c}\left|D_{x}^{2} \phi_{\varepsilon}\right|^{2} \mathrm{~d} x \\
& +c \int_{\mathbb{R}^{2}} \underbrace{\left|\int_{0}^{\infty} \rho_{\varepsilon} \chi_{1} \xi \mathrm{d} a\right|^{2}\left|\nabla_{x} \mathbf{v}\right|^{2}\left(Y_{\theta}^{\prime \prime}\right)^{2}}_{\leq c}\left|\nabla_{x} \phi_{\varepsilon}\right|^{4} \mathrm{~d} x+c \int_{\mathbb{R}^{2}} \underbrace{\left|\int_{0}^{\infty} \rho_{\varepsilon} \chi_{1} \xi \mathrm{d} a\right|^{2}\left|\nabla_{x} \mathbf{v}\right|^{2}\left(Y_{\theta}^{\prime}\right)^{2}}_{\leq c}\left|D_{x}^{2} \phi_{\varepsilon}\right|^{2} \mathrm{~d} x \\
& +c \int_{\mathbb{R}^{2}} \underbrace{\left|\int_{0}^{\infty} \rho_{\varepsilon} \chi_{1} \xi \mathrm{d} a\right|^{2}|\mathbf{v}|^{2}\left(Y_{\theta}^{\prime \prime \prime}\right)^{2}}_{\leq c}\left|\nabla_{x} \phi_{\varepsilon}\right|^{6} \mathrm{~d} x+c \int_{\mathbb{R}^{2}} \underbrace{\left|\int_{0}^{\infty} \rho_{\varepsilon} \chi_{1} \xi \mathrm{d} a\right|^{2}|\mathbf{v}|^{2}\left(Y_{\theta}^{\prime \prime}\right)^{2}}_{\leq c}\left|D_{x}^{2} \phi_{\varepsilon}\right|^{2}\left|\nabla_{x} \phi_{\varepsilon}\right|^{2} \mathrm{~d} x \\
& +c \int_{\mathbb{R}^{2}} \underbrace{\left|\int_{0}^{\infty} \rho_{\varepsilon} \chi 1 \xi \mathrm{d} a\right|^{2}|\mathbf{v}|^{2}\left(Y_{\theta}^{\prime}\right)^{2}}_{\leq c}\left|D_{x}^{3} \phi_{\varepsilon}\right|^{2} \mathrm{~d} x+c \int_{\mathbb{R}^{2}} \underbrace{\left|\nabla_{x} \mathfrak{d}\right|^{2}}_{\leq c} \cdot\left|\nabla_{x} \psi_{\varepsilon}\right|^{2} \mathrm{~d} x \\
& +c \int_{\mathbb{R}^{2}} \underbrace{\mathfrak{d}^{2}}_{\leq c}\left|\Delta_{x} \psi_{\varepsilon}\right|^{2} \mathrm{~d} x+c \int_{\mathbb{R}^{2}} \underbrace{\left|\Delta_{x} \mathfrak{d}\right|^{2}}_{\leq c}\left|\psi_{\varepsilon}\right|^{2} \mathrm{~d} x+c \int_{\mathbb{R}^{2}}|\int_{0}^{\infty} \nabla_{x} \rho_{\varepsilon} \cdot \nabla_{x} u_{\varepsilon} \xi \underbrace{\partial_{u} \mathfrak{p}\left(a, u_{\varepsilon}\right)}_{\leq c} \mathrm{~d} a|^{2} \mathrm{~d} x \\
& +c \int_{\mathbb{R}^{2}}\left|\int_{0}^{\infty} \Delta_{x} \rho_{\varepsilon} \xi \mathfrak{p}\left(a, u_{\varepsilon}\right) \mathrm{d} a\right|^{2} \mathrm{~d} x+\left.\left.c \int_{\mathbb{R}^{2}}|\underbrace{\int_{0}^{\infty} \rho_{\varepsilon}}_{\leq c_{T}} \xi \underbrace{\partial_{u u}^{2} \mathfrak{p}\left(a, u_{\varepsilon}\right)}_{\leq c}| \nabla_{x} u_{\varepsilon}\right|^{2} \mathrm{~d} a\right|^{2} \mathrm{~d} x \\
& +c \int_{\mathbb{R}^{2}}|\underbrace{\int_{0}^{\infty} \rho_{\varepsilon}}_{\leq c_{T}} \xi \underbrace{\partial_{u} \mathfrak{p}\left(a, u_{\varepsilon}\right)}_{\leq c} \Delta_{x} u_{\varepsilon} \mathrm{d} a|^{2} \mathrm{~d} x+c \int_{\mathbb{R}^{2}} \int_{0}^{\infty}\left|\Delta_{x} \rho_{\varepsilon}\right|^{2} \mathrm{~d} a \mathrm{~d} x \\
& \leq-\frac{\mu}{2} \int_{\mathbb{R}^{2}}\left|D_{x}^{4} \psi_{\varepsilon}\right|^{2} \mathrm{~d} x+c \int_{\mathbb{R}^{2}}\left|D_{x}^{3} \int_{0}^{\infty} \rho_{\varepsilon} \chi_{1} \xi \mathrm{d} a\right|^{2} \mathrm{~d} x+c \int_{\mathbb{R}^{2}}\left|D_{x}^{2} \int_{0}^{\infty} \rho_{\varepsilon} \chi_{1} \xi \mathrm{d} a\right|^{2} \mathrm{~d} x \\
& +c \int_{\mathbb{R}^{2}}\left|\nabla_{x} \int_{0}^{\infty} \rho_{\varepsilon} \chi_{1} \xi \mathrm{d} a\right|^{2} \mathrm{~d} x+c \int_{\mathbb{R}^{2}}\left|\int_{0}^{\infty} \rho_{\varepsilon} \chi_{1} \xi \mathrm{d} a\right|^{2} \mathrm{~d} x+c \int_{\mathbb{R}^{2}}\left|D_{x}^{2} \int_{0}^{\infty} \rho_{\varepsilon} \chi_{1} \xi \mathrm{d} a\right|^{4} \mathrm{~d} x \\
& +c \int_{\mathbb{R}^{2}}\left|\nabla_{x} \int_{0}^{\infty} \rho_{\varepsilon} \chi_{1} \xi \mathrm{d} a\right|^{4} \mathrm{~d} x+c \int_{\mathbb{R}^{2}}\left|D_{x}^{3} \phi_{\varepsilon}\right|^{2} \mathrm{~d} x+c \int_{\mathbb{R}^{2}}\left|D_{x}^{2} \phi_{\varepsilon}\right|^{2} \mathrm{~d} x+c \int_{\mathbb{R}^{2}}\left|D_{x}^{2} \phi_{\varepsilon}\right|^{4} \mathrm{~d} x \\
& +c \int_{\mathbb{R}^{2}}\left|\nabla_{x} \phi_{\varepsilon}\right|^{2} \mathrm{~d} x+c \int_{\mathbb{R}^{2}}\left|\nabla_{x} \phi_{\varepsilon}\right|^{4} \mathrm{~d} x+c \int_{\mathbb{R}^{2}}\left|\nabla_{x} \phi_{\varepsilon}\right|^{6} \mathrm{~d} x+c \int_{\mathbb{R}^{2}}\left|\nabla_{x} \phi_{\varepsilon}\right|^{8} \mathrm{~d} x \\
& +c \int_{\mathbb{R}^{2}} \psi_{\varepsilon}^{2} \mathrm{~d} x+c \int_{\mathbb{R}^{2}}\left|\nabla_{x} \psi_{\varepsilon}\right|^{2} \mathrm{~d} x+c \int_{\mathbb{R}^{2}}\left|D_{x}^{2} \psi_{\varepsilon}\right|^{2} \mathrm{~d} x+c \int_{\mathbb{R}^{2}}\left|\nabla_{x} \psi_{\varepsilon}\right|^{4} \mathrm{~d} x \\
& +c_{T} \int_{\mathbb{R}^{2}}\left|\nabla_{x} u_{\varepsilon}\right|^{4} \mathrm{~d} x+c_{T} \int_{\mathbb{R}^{2}}\left|\Delta_{x} u_{\varepsilon}\right|^{2} \mathrm{~d} x+c \int_{\mathbb{R}^{2}} \int_{0}^{\infty}\left|\Delta_{x} \rho_{\varepsilon}\right|^{2} \mathrm{~d} a \mathrm{~d} x .
\end{aligned}
$$

We integrate over $(0, t)$ and using the estimates (2.19), (2.18), 2.26), 2.27), 2.24), together with the embedding $H^{1}\left(\mathbb{R}^{2}\right) \subset L^{p}\left(\mathbb{R}^{2}\right)$ for very $1 \leq p<\infty$ give us

$$
\left\|D_{x}^{3} \psi_{\varepsilon}(t, \cdot)\right\|_{L^{2}\left(\mathbb{R}^{2}\right)}^{2}+\mu\left\|D_{x}^{4} \psi_{\varepsilon}\right\|_{L^{2}\left((0, t) \times \mathbb{R}^{2}\right)}^{2} \leq c e^{c t} .
$$


(2.30). It follows from (2.17), 2.18), 2.26), 2.27), 2.29), 2.31) and the embedding $H^{2}\left(\mathbb{R}^{2}\right) \subset$ $L^{\infty}\left(\mathbb{R}^{2}\right)$.

Lemma 2.9 (Estimates on the trace of $\rho_{\varepsilon}$ at $\left.a=0\right)$. Define $\rho_{\varepsilon}^{0}(t, x)=\rho_{\varepsilon}(t, 0, x)$. For every $t \geq$ 0 the following estimates hold

$$
\begin{aligned}
\left\|\rho_{\varepsilon}^{0}(t, \cdot)\right\|_{L^{1}\left(\mathbb{R}^{2}\right)},\left\|D_{x}^{2} \rho_{\varepsilon}^{0}\right\|_{L^{1}\left((0, t) \times \mathbb{R}^{2}\right)},\left\|\partial_{t} \rho_{\varepsilon}^{0}\right\|_{L^{1}\left((0, t) \times \mathbb{R}^{2}\right)} & \leq e^{C t} C, \\
\left\|\rho_{\varepsilon}^{0}(t, \cdot)\right\|_{L^{2}\left(\mathbb{R}^{2}\right)},\left\|\nabla_{x} \rho_{\varepsilon}^{0}(t, \cdot)\right\|_{L^{2}\left(\mathbb{R}^{2}\right)} & \leq e^{C t} C, \\
\left\|\nabla_{x} \rho_{\varepsilon}^{0}\right\|_{L^{2}\left((0, t) \times \mathbb{R}^{2}\right)},\left\|D_{x}^{2} \rho_{\varepsilon}^{0}\right\|_{L^{2}\left((0, t) \times \mathbb{R}^{2}\right)},\left\|\partial_{t} \rho_{\varepsilon}^{0}\right\|_{L^{2}\left((0, t) \times \mathbb{R}^{2}\right)} & \leq e^{C t} C, \\
\left\|\rho_{\varepsilon}^{0}(t, \cdot)\right\|_{L^{\infty}\left(\mathbb{R}^{2}\right)} & \leq e^{C t} C .
\end{aligned}
$$

Proof. Directly deduce from the definition of $\rho_{\varepsilon}$ at $a=0$, using Lemma 2.8, the estimates in (2.17), (2.18), and the assumptions on $\mathcal{A}(\phi)$ in (1.8).

Lemma $2.10\left(L^{\infty}\right.$ estimate on $\left.\rho_{\varepsilon}\right)$. We have that

$$
\left\|\rho_{\varepsilon}(t, \cdot, \cdot)\right\|_{L^{\infty}\left((0, \infty) \times \mathbb{R}^{2}\right)} \leq C e^{C t},
$$

for every $t \geq 0$.

Proof. Let $M$ be a positive constant that will be fixed later. Define

$$
\bar{\rho}_{\varepsilon}(t, a, x)=e^{-M t} \rho_{\varepsilon}(t, a, x) .
$$

$\bar{\rho}_{\varepsilon}$ satisfies the problem

$$
\left\{\begin{array}{l}
\partial_{t} \bar{\rho}_{\varepsilon}+M \bar{\rho}_{\varepsilon}+\partial_{a} \bar{\rho}_{\varepsilon}+\operatorname{div}_{x}\left(\bar{\rho}_{\varepsilon} \chi_{1}(a) \mathbf{v} Y_{\theta}\left(\phi_{\varepsilon}-R\right)\right)=\mu \Delta_{x} \bar{\rho}_{\varepsilon}+\varepsilon \partial_{a}\left(\chi(a) \partial_{a} \bar{\rho}_{\varepsilon}\right)-(\mathfrak{d}+\mathfrak{p}) \bar{\rho}_{\varepsilon} \\
\bar{\rho}_{\varepsilon}(t, 0, x)=\mathcal{A}\left(\phi_{\varepsilon}\right)\left(\int_{0}^{\infty} \bar{\rho}_{\varepsilon}(t, a, x) \chi_{3}(a) \mathrm{d} a\right) \omega(t, x) \\
\bar{\rho}_{\varepsilon}(0, a, x)=\rho_{0, \varepsilon}(a, x) .
\end{array}\right.
$$

Moreover, for any given $T>0$ there exists a sufficiently large constant $\mathcal{B}>0$ such that for any $t \leq T$ and $x \in \mathbb{R}^{2}$ (2.3), 2.11), and 2.9 imply

$$
0 \leq \bar{\rho}_{\varepsilon}(t, 0, x), \rho_{0, \varepsilon}(a, x) \leq \mathcal{B} .
$$

Consider

$$
\eta(\xi)=(\xi-\mathcal{B}) \mathbb{1}_{(\mathcal{B}, \infty)}(\xi)
$$

and observe that

$$
\eta^{\prime}(\xi)=\mathbb{1}_{(\mathcal{B}, \infty)}(\xi), \quad \xi \eta^{\prime}(\xi)=\eta(\xi)+\mathcal{B} \eta^{\prime}(\xi) .
$$

From the equation 2.33 we have

$$
\begin{aligned}
& \frac{d}{d t} \int_{0}^{\infty} \int_{\mathbb{R}^{2}} \eta\left(\bar{\rho}_{\varepsilon}\right) \mathrm{d} x \mathrm{~d} a=\int_{0}^{\infty} \int_{\mathbb{R}^{2}} \eta^{\prime}\left(\bar{\rho}_{\varepsilon}\right) \partial_{t} \bar{\rho}_{\varepsilon} \mathrm{d} x \mathrm{~d} a \\
& =-M \int_{0}^{\infty} \int_{\mathbb{R}^{2}} \eta^{\prime}\left(\bar{\rho}_{\varepsilon}\right) \bar{\rho}_{\varepsilon} \mathrm{d} x \mathrm{~d} a-\int_{0}^{\infty} \int_{\mathbb{R}^{2}} \eta^{\prime}\left(\bar{\rho}_{\varepsilon}\right) \partial_{a} \bar{\rho}_{\varepsilon} \mathrm{d} x \mathrm{~d} a-\int_{0}^{\infty} \int_{\mathbb{R}^{2}} \operatorname{div}_{x}\left(\bar{\rho}_{\varepsilon} \chi_{1} \mathbf{v} Y_{\theta}\right) \eta^{\prime}\left(\bar{\rho}_{\varepsilon}\right) \mathrm{d} x \mathrm{~d} a \\
& \quad+\mu \int_{0}^{\infty} \int_{\mathbb{R}^{2}} \eta^{\prime}\left(\bar{\rho}_{\varepsilon}\right) \Delta_{x} \bar{\rho}_{\varepsilon} \mathrm{d} x \mathrm{~d} a-\underbrace{\int_{0}^{\infty} \int_{\mathbb{R}^{2}} \eta^{\prime}\left(\bar{\rho}_{\varepsilon}\right)(\mathfrak{d}+\mathfrak{p}) \bar{\rho}_{\varepsilon} \mathrm{d} x \mathrm{~d} a}_{\leq 0}+\varepsilon \int_{0}^{\infty} \int_{\mathbb{R}^{2}} \eta^{\prime}\left(\bar{\rho}_{\varepsilon}\right) \partial_{a}\left(\chi(a) \partial_{a} \bar{\rho}_{\varepsilon}\right) \mathrm{d} x \mathrm{~d} a \\
& =\underbrace{-M \int_{0}^{\infty} \int_{\mathbb{R}^{2}} \eta\left(\bar{\rho}_{\varepsilon}\right) \mathrm{d} x \mathrm{~d} a}_{\leq 0}-M \mathcal{B} \int_{0}^{\infty} \int_{\mathbb{R}^{2}} \eta^{\prime}\left(\bar{\rho}_{\varepsilon}\right) \mathrm{d} x \mathrm{~d} a+\int_{\mathbb{R}^{2}} \eta\left(\bar{\rho}_{\varepsilon}(t, 0, x)\right) d x
\end{aligned}
$$




$$
\begin{aligned}
& -\int_{0}^{\infty} \int_{\mathbb{R}^{2}} \operatorname{div}_{x}\left(\left(\bar{\rho}_{\varepsilon}-\mathcal{B}\right) \chi_{1} \mathbf{v} Y_{\theta}\right) \eta^{\prime}\left(\bar{\rho}_{\varepsilon}\right) \mathrm{d} x \mathrm{~d} a \\
& -\mathcal{B} \int_{0}^{\infty} \int_{\mathbb{R}^{2}} \operatorname{div}_{x}\left(\chi_{1} \mathbf{v} Y_{\theta}\right) \eta^{\prime}\left(\bar{\rho}_{\varepsilon}\right) \mathrm{d} x \underbrace{\mathrm{d} a \underbrace{-\mu}_{0} \int_{0}^{\infty} \int_{\mathbb{R}^{2}} \eta^{\prime \prime}\left(\bar{\rho}_{\varepsilon}\right) \chi(a)\left(\nabla_{x} \bar{\rho}_{\varepsilon}\right)^{2} \mathrm{~d} x \mathrm{~d} a}_{\leq 0} \\
& -\varepsilon \int_{\mathbb{R}^{2}} \eta^{\prime}\left(\bar{\rho}_{\varepsilon}(t, 0, x)\right) \partial_{a} \bar{\rho}_{\varepsilon}(t, 0, x) d x \underbrace{-\varepsilon \int_{0}^{\infty} \int_{\mathbb{R}^{2}} \eta^{\prime \prime}\left(\bar{\rho}_{\varepsilon}\right)\left(\partial_{a} \bar{\rho}_{\varepsilon}\right)^{2} d a d x}_{=0} \\
& \leq \int_{\mathbb{R}^{2}} \eta\left(\bar{\rho}_{\varepsilon}(t, 0, x)\right) d x+\underbrace{\int_{0}^{\infty}}_{\int_{\mathbb{R}^{2}}\left(\bar{\rho}_{\varepsilon}-\mathcal{B}\right) \chi_{1}\left(\mathbf{v} \cdot \nabla_{x} \bar{\rho}_{\varepsilon}\right) Y_{\theta} \eta^{\prime \prime}\left(\bar{\rho}_{\varepsilon}\right) \mathrm{d} x \mathrm{~d} a} \\
& -\mathcal{B} \int_{0}^{\infty} \int_{\mathbb{R}^{2}}\left(M+\operatorname{div}_{x}\left(\chi_{1} \mathbf{v} Y_{\theta}\right)\right) \eta^{\prime}\left(\bar{\rho}_{\varepsilon}\right) \mathrm{d} x \mathrm{~d} a-\varepsilon \int_{\mathbb{R}^{2}} \eta^{\prime}\left(\bar{\rho}_{\varepsilon}(t, 0, x)\right) \partial_{a} \bar{\rho}_{\varepsilon}(t, 0, x) d x .
\end{aligned}
$$

Thanks to (2.9), (2.34), and (2.30) we have for $M$ large enough

$$
\eta\left(\bar{\rho}_{\varepsilon}(t, 0, x)\right)=0, \quad \eta^{\prime}\left(\bar{\rho}_{\varepsilon}(t, 0, x)\right)=0, \quad M+\operatorname{div}_{x}\left(\chi_{1} \mathbf{v} Y_{\theta}\right) \geq 0,
$$

which imply

$$
\frac{d}{d t} \int_{0}^{\infty} \int_{\mathbb{R}^{2}} \eta\left(\bar{\rho}_{\varepsilon}\right) \mathrm{d} x \mathrm{~d} a \leq 0 .
$$

Thus, we integrate over $(0, t)$ we obtain

$$
0 \leq \int_{0}^{\infty} \int_{\mathbb{R}^{2}} \eta\left(\bar{\rho}_{\varepsilon}(t, a, x)\right) \mathrm{d} x \mathrm{~d} a \leq \int_{0}^{\infty} \int_{\mathbb{R}^{2}} \eta\left(\rho_{0, \varepsilon}(a, x)\right) \mathrm{d} x \mathrm{~d} a=0,
$$

and

$$
\eta\left(\bar{\rho}_{\varepsilon}(t, a, x)\right)=0
$$

As a consequence we have that $\bar{\rho}_{\varepsilon} \leq \mathcal{B}$.

Im:11ro_a Lemma $2.11\left(L^{1}\right.$ estimate on $\left.\partial_{a} \rho_{\varepsilon}\right)$. We have that

$$
\left\|\partial_{a} \rho_{\varepsilon}(t, \cdot, \cdot)\right\|_{L^{1}\left((0, \infty) \times \mathbb{R}^{2}\right)} \leq C e^{C t}
$$

for every $t \geq 0$ and a suitable constant $C$ independent on $\varepsilon$.

Proof. Differentiating the equation in (2.14) with respect to $a$ we get

$$
\begin{aligned}
\partial_{t a}^{2} \rho_{\varepsilon} & +\partial_{a a}^{2} \rho_{\varepsilon}+\operatorname{div}_{x}\left(\partial_{a} \rho_{\varepsilon} \chi_{1} \mathbf{v} Y_{\theta}\right)+\operatorname{div}_{x}\left(\rho_{\varepsilon} \chi_{1}^{\prime} \mathbf{v} Y_{\theta}\right) \\
& =\mu \Delta_{x} \partial_{a} \rho_{\varepsilon}+\varepsilon \partial_{a a}^{2}\left(\chi(a) \partial_{a} \rho_{\varepsilon}\right)-\left(\partial_{a} \mathfrak{d}+\partial_{a} \mathfrak{p}\left(a, u_{\varepsilon}\right)\right) \rho_{\varepsilon}-(\mathfrak{d}+\mathfrak{p}) \partial_{a} \rho_{\varepsilon} .
\end{aligned}
$$

Then

$$
\begin{aligned}
& \frac{d}{d t} \int_{0}^{\infty} \int_{\mathbb{R}^{2}}\left|\partial_{a} \rho_{\varepsilon}\right| \mathrm{d} x \mathrm{~d} a=\int_{0}^{\infty} \int_{\mathbb{R}^{2}} \partial_{t a}^{2} \rho_{\varepsilon} \operatorname{sign}\left(\partial_{a} \rho_{\varepsilon}\right) \mathrm{d} x \mathrm{~d} a \\
& =\int_{0}^{\infty} \int_{\mathbb{R}^{2}}\left(\varepsilon \partial_{a a}^{2}\left(\chi(a) \partial_{a} \rho_{\varepsilon}\right)-\partial_{a a}^{2} \rho_{\varepsilon}\right) \operatorname{sign}\left(\partial_{a} \rho_{\varepsilon}\right) \mathrm{d} x \mathrm{~d} a \underbrace{+\mu \int_{0}^{\infty} \int_{\mathbb{R}^{2}} \Delta_{x} \partial_{a} \rho_{\varepsilon} \operatorname{sign}\left(\partial_{a} \rho_{\varepsilon}\right) \mathrm{d} x \mathrm{~d} a}_{=0} \\
& \underbrace{-\int_{0}^{\infty} \int_{\mathbb{R}^{2}} \operatorname{div}_{x}\left(\partial_{a} \rho_{\varepsilon} \chi_{1} \mathbf{v} Y_{\theta}\right) \operatorname{sign}\left(\partial_{a} \rho_{\varepsilon}\right) \mathrm{d} x \mathrm{~d} a}_{=0}-\int_{0}^{\infty} \int_{\mathbb{R}^{2}} \nabla_{x} \rho_{\varepsilon} \cdot \mathbf{v} \chi_{1}^{\prime} Y_{\theta} \operatorname{sign}\left(\partial_{a} \rho_{\varepsilon}\right) \mathrm{d} x \mathrm{~d} a \\
& -\int_{0}^{\infty} \int_{\mathbb{R}^{2}} \rho_{\varepsilon} \chi_{1}^{\prime} \operatorname{div}_{x}(\mathbf{v}) Y_{\theta} \operatorname{sign}\left(\partial_{a} \rho_{\varepsilon}\right) \mathrm{d} x \mathrm{~d} a-\int_{0}^{\infty} \int_{\mathbb{R}^{2}} \rho_{\varepsilon} \chi_{1}^{\prime} \mathbf{v} \cdot \nabla_{x} \phi_{\varepsilon} Y_{\theta}^{\prime} \operatorname{sign}\left(\partial_{a} \rho_{\varepsilon}\right) \mathrm{d} x \mathrm{~d} a
\end{aligned}
$$




$$
\begin{aligned}
& \quad-\int_{0}^{\infty} \int_{\mathbb{R}^{2}} \underbrace{\left(\partial_{a} \mathfrak{d}+\partial_{a}\left(\mathfrak{p}\left(a, u_{\varepsilon}\right)\right)\right)}_{\leq c} \rho_{\varepsilon} \operatorname{sign}\left(\partial_{a} \rho_{\varepsilon}\right) \mathrm{d} x \mathrm{~d} a \underbrace{-\underbrace{\infty}_{0} \int_{\mathbb{R}^{2}}(\mathfrak{d}+\mathfrak{p})\left|\partial_{a} \rho_{\varepsilon}\right| \mathrm{d} x \mathrm{~d} a}_{\leq 0} \\
& \leq \int_{\mathbb{R}^{2}}\left|\varepsilon \partial_{a}\left(\chi(0) \partial_{a} \rho_{\varepsilon}(t, 0, x)\right)-\partial_{a} \rho_{\varepsilon}(t, 0, x)\right| d x+c\left\|\nabla_{x} \rho_{\varepsilon}(t, \cdot, \cdot)\right\|_{L^{2}\left((0, \infty) \times \mathbb{R}^{2}\right)}\|\mathbf{v}\|_{L^{2}\left(\mathbb{R}^{2}\right)} \\
& \quad+c\left\|\rho_{\varepsilon}(t, \cdot, \cdot)\right\|_{L^{2}\left((0, \infty) \times \mathbb{R}^{2}\right)}\left\|\operatorname{div}_{x}(\mathbf{v})\right\|_{L^{2}\left(\mathbb{R}^{2}\right)}+c\left\|\rho_{\varepsilon}(t, \cdot, \cdot)\right\|_{L^{2}\left((0, \infty) \times \mathbb{R}^{2}\right)}\left\|\nabla_{x} \phi_{\varepsilon}(t, \cdot)\right\|_{L^{2}\left(\mathbb{R}^{2}\right)} \\
& \quad+c\left\|\rho_{\varepsilon}(t, \cdot, \cdot)\right\|_{L^{1}\left((0, \infty) \times \mathbb{R}^{2}\right)} .
\end{aligned}
$$

Since

$$
\varepsilon \partial_{a}\left(\chi(0) \partial_{a} \rho_{\varepsilon}(t, 0, x)\right)-\partial_{a} \rho_{\varepsilon}(t, 0, x)=\partial_{t} \rho_{\varepsilon}^{0}-\mu \Delta_{x} \rho_{\varepsilon}^{0}+\mathfrak{d} \rho_{\varepsilon}^{0},
$$

we use the Gronwall Lemma, (2.3), (2.17), 2.26), (2.12), 2.13), 2.19), (2.6) and Lemma 2.9 to obtain the inequality 2.35.

Lemma 2.12 (BV estimate w.r.t. $x$ on $u_{\varepsilon}$ ). For every $t \geq 0$ the following estimate holds

$$
\left\|\nabla_{x} u_{\varepsilon}(t, \cdot)\right\|_{L^{1}\left(\mathbb{R}^{2}\right)} \leq C e^{C t} .
$$

Proof. From the equation of $u_{\varepsilon}$ in 2.25 we have the equation

$$
\partial_{t} \nabla_{x} u_{\varepsilon}+\nabla_{x}\left(\nabla u_{\varepsilon} \cdot \nu\left(\phi_{\varepsilon}\right)+u_{\varepsilon} \operatorname{div}_{x}\left(\nu\left(\phi_{\varepsilon}\right)\right)\right)=\left(\mathfrak{b}\left(\phi_{\varepsilon}\right)-\beta\right) \nabla_{x} u_{\varepsilon}+\mathfrak{b}^{\prime}\left(\phi_{\varepsilon}\right) \nabla_{x} \phi_{\varepsilon} u_{\varepsilon}+\varepsilon \nabla_{x} \Delta_{x} u_{\varepsilon} .
$$

We define

$$
\operatorname{sign}\left(\nabla_{x} u_{\varepsilon}\right)=\left(\operatorname{sign}\left(\partial_{x_{1}}\left(u_{\varepsilon}\right)\right), \operatorname{sign}\left(\partial_{x_{2}}\left(u_{\varepsilon}\right)\right)\right)
$$

where $x=\left(x_{1}, x_{2}\right)$. Then, using the $L^{\infty}$ bounds on $\nabla_{x} \phi_{\varepsilon}$ and $\nu\left(\phi_{\varepsilon}\right)$ in 2.30, 2.5), we get

$$
\begin{aligned}
& \frac{d}{d t} \int_{\mathbb{R}^{2}}\left|\nabla_{x} u_{\varepsilon}\right| \mathrm{d} x=\int_{\mathbb{R}^{2}} \operatorname{sign}\left(\nabla_{x} u_{\varepsilon}\right) \partial_{t} \nabla_{x} u_{\varepsilon} \mathrm{d} x \\
& =-\underbrace{\int_{\mathbb{R}^{2}} \nabla_{x}\left(\nabla_{x} u_{\varepsilon} \cdot \nu\left(\phi_{\varepsilon}\right)\right) \operatorname{sign}\left(\nabla_{x} u_{\varepsilon}\right) \mathrm{d} x}_{=0}-\int_{\mathbb{R}^{2}} \nabla_{x}\left(u_{\varepsilon} \operatorname{div}_{x}\left(\nu\left(\phi_{\varepsilon}\right)\right)\right) \operatorname{sign}\left(\nabla_{x} u_{\varepsilon}\right) \mathrm{d} x \\
& \quad+\underbrace{\varepsilon \int_{\mathbb{R}^{2}} \nabla_{x} \Delta_{x} u_{\varepsilon} \operatorname{sign}\left(\nabla_{x} u_{\varepsilon}\right) \mathrm{d} x}_{\leq 0}+\int_{\mathbb{R}^{2}} \underbrace{\mathfrak{b}^{\prime}\left(\phi_{\varepsilon}\right) \nabla_{x} \phi_{\varepsilon}}_{\leq c_{T}} u_{\varepsilon} \operatorname{sign}\left(\nabla_{x} u_{\varepsilon}\right) \mathrm{d} x+\int_{\mathbb{R}^{2}} \underbrace{\left(\mathfrak{b}\left(\phi_{\varepsilon}\right)-\beta\right)}_{\leq c}\left|\nabla_{x} u_{\varepsilon}\right| \mathrm{d} x \\
& \leq \int_{\mathbb{R}^{2}}\left|\nabla_{x} u_{\varepsilon}\right|\left|\operatorname{div}_{x}\left(\nu\left(\phi_{\varepsilon}\right)\right)\right| \mathrm{d} x-\int_{\mathbb{R}^{2}} u_{\varepsilon} \nabla_{x}\left(\operatorname{div}_{x}\left(\nu\left(\phi_{\varepsilon}\right)\right)\right) \operatorname{sign}\left(\nabla_{x} u_{\varepsilon}\right) \mathrm{d} x \\
& \quad+c_{T} \int_{\mathbb{R}^{2}} u_{\varepsilon} \mathrm{d} x+c \int_{\mathbb{R}^{2}}\left|\nabla_{x} u_{\varepsilon}\right| \mathrm{d} x \\
& \leq c \int_{\mathbb{R}^{2}}\left|\nabla_{x} u_{\varepsilon}\right| \mathrm{d} x+c_{T} \int_{\mathbb{R}^{2}}\left|u_{\varepsilon}\right| \mathrm{d} x+c \int_{\mathbb{R}^{2}} u_{\varepsilon}^{2} \mathrm{~d} x+c \int_{\mathbb{R}^{2}}\left|\nabla_{x} u_{\varepsilon}\right|^{2} \mathrm{~d} x \\
& \quad+\int_{\mathbb{R}^{2}}\left|\operatorname{div}_{x}\left(\nu\left(\phi_{\varepsilon}\right)\right)\right|^{2} \mathrm{~d} x+\int_{\mathbb{R}^{2}}\left|\nabla_{x}\left(\operatorname{div}_{x}\left(\nu\left(\phi_{\varepsilon}\right)\right)\right)\right|^{2} \mathrm{~d} x .
\end{aligned}
$$

We obtain the desired inequality (2.36) integrating over $(0, t)$, using the Gronwall Lemma and the estimates in (2.6) and 2.9) and Lemma 2.7 .

BV in $\mathrm{t}$ Lemma 2.13 (BV estimate w.r.t. $t$ on $u_{\varepsilon}$ ). For every $t \geq 0$, the following estimate holds

$$
\left\|\partial_{t} u_{\varepsilon}(t, \cdot)\right\|_{L^{1}\left(\mathbb{R}^{2}\right)} \leq C e^{C t} .
$$


Proof. From the definition of $\nu(\phi)$ in 1.2 we compute

$$
\partial_{t} \nu\left(\phi_{\varepsilon}\right)=\kappa \frac{\partial_{t} \phi_{\varepsilon} * \nabla_{x} \eta}{\left(1+\left\|\phi_{\varepsilon} * \nabla_{x} \eta\right\|^{2}\right)^{3 / 2}}
$$

then from 2.30 we have

$$
\left\|\partial_{t} \nu\left(\phi_{\varepsilon}\right)\right\|_{L^{\infty}\left(\mathbb{R}^{2} ; \mathbb{R}^{2}\right)} \leq \kappa\left\|\nabla_{x} \eta\right\|_{L^{\infty}\left(\mathbb{R}^{2} ; \mathbb{R}^{2}\right)}\left\|\partial_{t} \phi_{\varepsilon}\right\|_{L^{1}\left(\mathbb{R}^{2}, \mathbb{R}\right)} \leq C e^{C t}
$$

Similarly, explicit computations give us

$$
\operatorname{div}_{x}\left(\nu\left(\phi_{\varepsilon}\right)\right)=\kappa \frac{\phi_{\varepsilon} * \Delta_{x} \eta}{\left(1+\left\|\phi_{\varepsilon} * \nabla_{x} \eta\right\|^{2}\right)^{3 / 2}}
$$

and

$$
\partial_{t} \operatorname{div}_{x}\left(\nu\left(\phi_{\varepsilon}\right)\right)=\kappa \frac{\partial_{t} \phi_{\varepsilon} * \Delta_{x} \eta}{\left(1+\left\|\phi_{\varepsilon} * \nabla_{x} \eta\right\|^{2}\right)^{3 / 2}}-3 \kappa\left(\phi_{\varepsilon} * \Delta_{x} \eta\right) \frac{\phi_{\varepsilon} * \nabla_{x} \eta}{\sqrt{1+\left\|\phi_{\varepsilon} * \nabla_{x} \eta\right\|^{2}}} \frac{\partial_{t} \phi_{\varepsilon} * \nabla_{x} \eta}{\left(1+\left\|\phi_{\varepsilon} * \nabla_{x} \eta\right\|^{2}\right)^{2}} .
$$

Therefore, from (2.17), 2.30), we obtain

$$
\begin{aligned}
& \left\|\partial_{t} \operatorname{div}_{x}\left(\nu\left(\phi_{\varepsilon}\right)\right)\right\|_{L^{2}\left(\mathbb{R}^{2} ; \mathbb{R}\right)} \\
& \quad \leq \kappa\left\|\partial_{t} \phi_{\varepsilon} * \Delta_{x} \eta\right\|_{L^{2}\left(\mathbb{R}^{2} ; \mathbb{R}\right)}+3 \kappa\left\|\phi_{\varepsilon} * \Delta_{x} \eta\right\|_{L^{\infty}\left(\mathbb{R}^{2} ; \mathbb{R}\right)}\left\|\partial_{t} \phi_{\varepsilon} * \nabla_{x} \eta\right\|_{L^{2}\left(\mathbb{R}^{2} ; \mathbb{R}^{2}\right)} \\
& \quad \leq \kappa\left\|\partial_{t} \phi_{\varepsilon}\right\|_{L^{1}\left(\mathbb{R}^{2} ; \mathbb{R}\right)}\left(\left\|\Delta_{x} \eta\right\|_{L^{2}\left(\mathbb{R}^{2} ; \mathbb{R}\right)}+3\left\|\Delta_{x} \eta\right\|_{L^{\infty}\left(\mathbb{R}^{2} ; \mathbb{R}\right)}\left\|\nabla_{x} \eta\right\|_{L^{2}\left(\mathbb{R}^{2} ; \mathbb{R}^{2}\right)}\left\|\phi_{\varepsilon}\right\|_{L^{1}\left(\mathbb{R}^{2} ; \mathbb{R}\right)}\right) \\
& \quad \leq C e^{C t}
\end{aligned}
$$

The estimates above allow us to prove (2.37). From the equation 2.25) for $u_{\varepsilon}$ we have

$$
\partial_{t t}^{2} u_{\varepsilon}+\operatorname{div}_{x}\left(\partial_{t} u_{\varepsilon} \nu\left(\phi_{\varepsilon}\right)\right)+\operatorname{div}_{x}\left(u_{\varepsilon} \partial_{t} \nu\left(\phi_{\varepsilon}\right)\right)=\left(\mathfrak{b}\left(\phi_{\varepsilon}\right)-\beta\right) \partial_{t} u_{\varepsilon}+\mathfrak{b}^{\prime}\left(\phi_{\varepsilon}\right) \partial_{t} \phi_{\varepsilon} u_{\varepsilon}+\varepsilon \Delta_{x} \partial_{t} u_{\varepsilon},
$$

then we consider

$$
\begin{aligned}
\frac{d}{d t} \int_{\mathbb{R}^{2}}\left|\partial_{t} u_{\varepsilon}\right| \mathrm{d} x= & -\underbrace{\int_{\mathbb{R}^{2}} \operatorname{div}_{x}\left(\partial_{t} u_{\varepsilon} \nu\left(\phi_{\varepsilon}\right)\right) \operatorname{sign}\left(\partial_{t} u_{\varepsilon}\right) \mathrm{d} x}_{=0}+\varepsilon \underbrace{\int_{\mathbb{R}^{2}} \Delta_{x} \partial_{t} u_{\varepsilon} \operatorname{sign}\left(\partial_{t} u_{\varepsilon}\right) \mathrm{d} x}_{\leq 0} \\
& -\int_{\mathbb{R}^{2}}(\nabla u_{\varepsilon} \underbrace{\partial_{t} \nu\left(\phi_{\varepsilon}\right)}_{\leq c_{T}}+u_{\varepsilon} \partial_{t} \operatorname{div}_{x}\left(\nu\left(\phi_{\varepsilon}\right)\right)) \operatorname{sign}\left(\partial_{t} u_{\varepsilon}\right) \mathrm{d} x \\
& +\int_{\mathbb{R}^{2}} \underbrace{\left(\mathfrak{b}\left(\phi_{\varepsilon}\right)-\beta\right)}_{\leq c}\left|\partial_{t} u_{\varepsilon}\right| \mathrm{d} x+\int_{\mathbb{R}^{2}} \mathfrak{b}^{\prime}\left(\phi_{\varepsilon}\right) \partial_{t} \phi_{\varepsilon} u_{\varepsilon} \operatorname{sign}\left(\partial_{t} u_{\varepsilon}\right) \mathrm{d} x \\
\leq & c_{T} \int_{\mathbb{R}^{2}}\left|\nabla_{x} u_{\varepsilon}\right| \mathrm{d} x+c \int_{\mathbb{R}^{2}} u_{\varepsilon}^{2} \mathrm{~d} x+\int_{\mathbb{R}^{2}}\left|\partial_{t} \operatorname{div}_{x}\left(\nu\left(\phi_{\varepsilon}\right)\right)\right|^{2} d x \\
& +c_{T} \int_{\mathbb{R}^{2}}\left|\partial_{t} \phi_{\varepsilon}\right| \mathrm{d} x+c \int_{\mathbb{R}^{2}}\left|\partial_{t} u_{\varepsilon}\right| \mathrm{d} x .
\end{aligned}
$$

Integrating on $(0, t)$ we obtain 2.37) thanks to 2.23, 2.30 and 2.36) and the Gronwall Lemma.

We are now ready to prove the compactness of the families $\left\{\rho_{\varepsilon}\right\}_{\varepsilon}$ and $\left\{u_{\varepsilon}\right\}_{\varepsilon}$, and the first part of Theorem 1.1, establishing the existence of entropy solutions for the system (1.1). 
lem:exist Lemma 2.14 (Strong compactness of $\left\{\rho_{\varepsilon}\right\}_{\varepsilon}$ and $\left\{u_{\varepsilon}\right\}_{\varepsilon}$ ). There exists a couple of functions $(u, \rho)$ and a sequence $\left\{\varepsilon_{k}\right\}_{k \in \mathbb{N}} \in(0, \infty), \varepsilon_{k} \rightarrow 0$, such that, for every $T>0$

$$
\begin{aligned}
& \rho_{\varepsilon_{k}} \rightarrow \rho, \quad \text { a.e. in }(0, T) \times(0, \infty) \times \mathbb{R}^{2} \quad \text { and in } L_{\text {loc }}^{p}\left((0, \infty) \times(0, \infty) \times \mathbb{R}^{2}\right), 1 \leq p<\infty, \\
& \rho(\cdot, \cdot, \cdot) \geq 0, \quad \rho(t, \cdot, x) \in B V(0, \infty), \quad \text { for a.e. }(t, x) \in(0, \infty) \times \mathbb{R}^{2}, \\
& \rho \in L^{\infty}\left(0, T ; L^{1}\left((0, \infty) \times \mathbb{R}^{2}\right)\right) \cap L^{\infty}\left((0, T) \times(0, \infty) \times \mathbb{R}^{2}\right) \cap L^{2}\left((0, T) \times(0, \infty) ; H^{2}\left(\mathbb{R}^{2}\right)\right),
\end{aligned}
$$

and

$$
\begin{aligned}
& u_{\varepsilon_{k_{h}}} \rightarrow u \quad \text { a.e. in }(0, T) \times \mathbb{R}^{2} \quad \text { and in } L_{\mathrm{loc}}^{p}\left((0, \infty) \times \mathbb{R}^{2}\right), 1 \leq p<\infty, \\
& u(\cdot, \cdot) \geq 0, \quad u \in L^{\infty}\left((0, T) \times \mathbb{R}^{2}\right) \cap B V\left((0, T) \times \mathbb{R}^{2}\right) .
\end{aligned}
$$

Proof. Rewrite equation of $\rho_{\varepsilon}$ in 2.14

$$
\begin{aligned}
\partial_{t} \rho_{\varepsilon}+\operatorname{div}_{(a, x)}\left(\begin{array}{c}
\rho_{\varepsilon}^{2} / 2 \\
\rho_{\varepsilon}^{2} / 2
\end{array}\right) & =\left(\rho_{\varepsilon}-1\right) \partial_{a} \rho_{\varepsilon}+\rho_{\varepsilon} \operatorname{div}_{x}\left(\rho_{\varepsilon}\right) \\
& -\operatorname{div}_{x}\left(\rho_{\varepsilon} \chi_{1}(a) \mathbf{v} Y_{\theta}\left(\phi_{\varepsilon}-R\right)\right)+\mu \Delta_{x} \rho_{\varepsilon}+\varepsilon \partial_{a}\left(\chi(a) \partial_{a} \rho_{\varepsilon}\right)-(\mathfrak{d}+\mathfrak{p}) \rho_{\varepsilon} .
\end{aligned}
$$

Let $\eta \in C^{2}\left(\mathbb{R}^{2}\right)$ be a convex entropy with flux $Q \in C^{2}\left(\mathbb{R} ; \mathbb{R}^{2}\right)$ such that

$$
Q^{\prime}(\rho)=\left(\begin{array}{l}
\rho \eta^{\prime}(\rho) \\
\rho \eta^{\prime}(\rho)
\end{array}\right) .
$$

Multiplying both sides of 2.41) by $\eta^{\prime}\left(\rho_{\varepsilon}\right)$ we get

$$
\begin{aligned}
\partial_{t} \eta\left(\rho_{\varepsilon}\right) & +\operatorname{div}_{(a, x)}\left(Q\left(\rho_{\varepsilon}\right)\right) \\
= & \underbrace{\eta^{\prime}\left(\rho_{\varepsilon}\right)\left[\left(\rho_{\varepsilon}-1\right) \partial_{a} \rho_{\varepsilon}+\rho_{\varepsilon} \operatorname{div}_{x}\left(\rho_{\varepsilon}\right)-\operatorname{div}_{x}\left(\rho_{\varepsilon} \chi \chi_{1}(a) \mathbf{v} Y_{\theta}\left(\phi_{\varepsilon}-R\right)\right)+\mu \Delta_{x} \rho_{\varepsilon}-(\mathfrak{d}+\mathfrak{p}) \rho_{\varepsilon}\right]}_{\mathcal{L}_{1, \varepsilon}} \\
& +\underbrace{\partial_{a}\left(\varepsilon \eta^{\prime}\left(\rho_{\varepsilon}\right) \chi(a) \partial_{a} \rho_{\varepsilon}\right)}_{\mathcal{L}_{2, \varepsilon}}-\underbrace{\varepsilon \eta^{\prime \prime}\left(\rho_{\varepsilon}\right) \chi(a)\left(\partial_{a} \rho_{\varepsilon}\right)^{2}}_{\mathcal{L}_{3, \varepsilon}} .
\end{aligned}
$$

For every $K \subset \subset(0, \infty) \times(0, \infty) \times \mathbb{R}^{2}$, thanks to Lemmas 2.3, 2.4, 2.5, 2.8, 2.10, 2.11 and the estimates in (2.17), 2.18), we have

$$
\begin{aligned}
\left\|\mathcal{L}_{1, \varepsilon}\right\|_{L^{1}(K)} \leq & \left\|\eta^{\prime}\left(\rho_{\varepsilon}\right)\right\|_{L^{\infty}(K)}\left[\left(\left\|\rho_{\varepsilon}\right\|_{L^{\infty}(K)}+1\right)\left\|\partial_{a} \rho_{\varepsilon}\right\|_{L^{1}(K)}+\left\|\rho_{\varepsilon}\right\|_{L^{2}(K)}\left\|\nabla_{x} \rho_{\varepsilon}\right\|_{L^{2}(K)}\right. \\
& +c\left\|\rho_{\varepsilon}\right\|_{L^{2}(K)}\left\|\nabla_{x} \phi_{\varepsilon}\right\|_{L^{2}(K)}+c\left\|\phi_{\varepsilon}\right\|_{L^{2}(K)}\left\|\rho_{\varepsilon}\right\|_{L^{2}(K)} \\
& \left.+c\left\|\phi_{\varepsilon}\right\|_{L^{2}(K)}\left\|\nabla_{x} \rho_{\varepsilon}\right\|_{L^{2}(K)}+c\left\|\Delta_{x} \rho_{\varepsilon}\right\|_{L^{2}(K)}+\|\mathfrak{d}\|_{L^{\infty}(K)}\left\|\rho_{\varepsilon}\right\|_{L^{1}(K)}\right] \leq c, \\
\left\|\varepsilon \eta^{\prime}\left(\rho_{\varepsilon}\right) \chi(a) \partial_{a} \rho_{\varepsilon}\right\|_{L^{2}(K)} \leq & \varepsilon\left\|\eta^{\prime}\left(\rho_{\varepsilon}\right)\right\|_{L^{\infty}(K)}\left\|\chi(a) \partial_{a} \rho_{\varepsilon}\right\|_{L^{2}(K)} \leq c \sqrt{\varepsilon} \rightarrow 0, \\
\left\|\mathcal{L}_{3, \varepsilon}\right\|_{L^{1}(K)} \leq & \varepsilon\left\|\eta^{\prime \prime}\left(\rho_{\varepsilon}\right)\right\|_{L^{\infty}(K)}\left\|\chi(a) \partial_{a} \rho_{\varepsilon}^{2}\right\|_{L^{1}(K)} \leq c .
\end{aligned}
$$

Applying the Murat Lemma in [9] to get

$$
\left\{\partial_{t} \eta\left(\rho_{\varepsilon}\right)+\operatorname{div}_{(a, x)}\left(Q\left(\rho_{\varepsilon}\right)\right)\right\}_{\varepsilon} \text { is compact in } H_{l o c}^{-1}\left((0, \infty) \times(0, \infty) \times \mathbb{R}^{2}\right),
$$

this implies the strong compactness of $\left\{\rho_{\varepsilon}\right\}_{\varepsilon}$.

Thanks to Lemmas 2.21, 2.13, 2.12, $\left\{u_{\varepsilon}\right\}_{\varepsilon}$ is bounded in $L^{\infty}\left((0, T) \times \mathbb{R}^{2}\right) \cap B V\left((0, T) \times \mathbb{R}^{2}\right)$ so that Helly's Theorem applies.

Lemma 2.15. The couple of functions $(u, \rho)$ introduced in Lemma 2.14 is an entropy solution of (1.1) in the sense of Definition 1.2 . 
Proof. It is clear that the couple $(u, \rho)$ is a weak solution of (1.1) in the sense of Definition 1.1 thanks to the strong convergence results in Lemma 2.14. In particular, the fact that $\rho$ is a weak solution comes directly from [11, Th. 5].

We obtain (1.14) as in [2, Lemma 2.10], then we only have to verify that 1.15) holds. Let $\xi \in$ $C^{\infty}\left(\mathbb{R}^{4}\right)$ be a nonnegative text function with compact support and $\mathfrak{c} \in \mathbb{R}$ be a constant. Multiplying the equation of $u_{\varepsilon}$ in (2.1) by $\operatorname{sign}\left(u_{\varepsilon}-\mathfrak{c}\right)$ we have

$$
\begin{aligned}
\partial_{t}\left|u_{\varepsilon}-\mathfrak{c}\right|+\operatorname{div}_{x}\left(\left|u_{\varepsilon}-\mathfrak{c}\right| \nu\left(\phi_{\varepsilon}\right)\right) & +\mathfrak{c} \operatorname{sign}\left(u_{\varepsilon}-\mathfrak{c}\right) \operatorname{div}_{x}\left(\nu\left(\phi_{\varepsilon}\right)\right) \\
& \leq \operatorname{sign}\left(u_{\varepsilon}-\mathfrak{c}\right)\left(\mathfrak{b}\left(\phi_{\varepsilon}\right)-\beta\right) u_{\varepsilon}+\varepsilon \Delta_{x}\left|u_{\varepsilon}-\mathfrak{c}\right| .
\end{aligned}
$$

Then,

$$
\begin{gathered}
\int_{0}^{\infty} \int_{0}^{\infty} \int_{\mathbb{R}^{2}}\left(\left|u_{\varepsilon}-\mathfrak{c}\right| \partial_{t} \xi+\left|u_{\varepsilon}-\mathfrak{c}\right| \nu\left(\phi_{\varepsilon}\right) \cdot \nabla_{x} \xi+\operatorname{sign}\left(u_{\varepsilon}-\mathfrak{c}\right)\left(\mathfrak{b}\left(\phi_{\varepsilon}\right)-\beta\right) u_{\varepsilon} \xi\right. \\
\left.+\varepsilon\left|u_{\varepsilon}-\mathfrak{c}\right| \Delta_{x} \xi-\mathfrak{c} \operatorname{sign}\left(u_{\varepsilon}-\mathfrak{c}\right) \operatorname{div}_{x}\left(\nu\left(\phi_{\varepsilon}\right)\right) \xi\right) \mathrm{d} x \mathrm{~d} a \mathrm{~d} t \\
+\int_{0}^{\infty} \int_{\mathbb{R}^{2}}\left|u_{\varepsilon, 0}(x)-\mathfrak{c}\right| \xi(0, a, x) \mathrm{d} x \mathrm{~d} a \geq 0 .
\end{gathered}
$$

By taking the limit for $\varepsilon \rightarrow 0$, we get (1.15).

\section{UNIQUENESS AND STABILITY}

In this section we establish the inequality (1.16), which concludes the proof of Theorem 1.1. To this end we introduce the following preliminary lemma.

:doubling Lemma 3.1. Let $\left(u_{1}, \rho_{1}\right)$ and $\left(u_{2}, \rho_{2}\right)$ be two entropy solutions of (1.1) obtained from the initial data $\left(u_{1,0}, \rho_{1,0}\right)$ and $\left(u_{2,0}, \rho_{2,0}\right)$ respectively. For every nonnegative test function $\xi \in C_{c}^{\infty}\left(\mathbb{R}^{4}\right)$ the following inequalities hold

$$
\begin{aligned}
& \int_{0}^{\infty} \int_{0}^{\infty} \int_{\mathbb{R}^{2}}\left(\left|\rho_{1}-\rho_{2}\right|\left(\partial_{t} \xi+\partial_{a} \xi\right)-\operatorname{div}_{x}\left(\left|\rho_{1}-\rho_{2}\right| \chi_{1} \mathbf{v}\left(Y_{\theta}\left(\phi_{1}-R\right)+Y_{\theta}\left(\phi_{2}-R\right)\right)\right) \xi\right. \\
& +\mu \Delta_{x}\left|\rho_{1}-\rho_{2}\right| \xi-\left|\rho_{1}-\rho_{2}\right| \mathfrak{d} \xi-\left|\rho_{1}-\rho_{2}\right| \mathfrak{p}\left(a, u_{1}\right) \xi \\
& \left.\quad-\operatorname{sign}\left(\rho_{1}-\rho_{2}\right)\left(\mathfrak{p}\left(a, u_{1}\right)-\mathfrak{p}\left(a, u_{2}\right)\right) \rho_{2} \xi\right) d x d a d t \\
& +\int_{0}^{\infty} \int_{\mathbb{R}^{2}}\left|\rho_{1}\left(t, 0^{+}, x\right)-\rho_{2}\left(t, 0^{+}, x\right)\right| \xi(t, 0, x) d x d t+\int_{0}^{\infty} \int_{\mathbb{R}^{2}}\left|\rho_{1,0}(a, x)-\rho_{2,0}(a, x)\right| \xi(0, a, x) d x d a \\
& \geq \int_{0}^{\infty} \int_{0}^{\infty} \int_{\mathbb{R}^{2}} \operatorname{sign}\left(\rho_{1}-\rho_{2}\right) \chi_{1}\left(\rho_{2} \operatorname{div}_{x}\left(\mathbf{v} Y_{\theta}\left(\phi_{1}-R\right)\right)-\rho_{1} \operatorname{div}_{x}\left(\mathbf{v}(x) Y_{\theta}\left(\phi_{2}-R\right)\right)\right) \xi d x d a d t
\end{aligned}
$$

where

$$
\phi_{i}(t, x):=\int_{0}^{\infty} \rho_{i}(t, a, x) \chi_{2}(a) d a, \quad \text { for } i=1,2
$$

and

$$
\begin{aligned}
& \int_{0}^{\infty} \int_{0}^{\infty} \int_{\mathbb{R}^{2}}\left(\left|u_{1}-u_{2}\right| \partial_{t} \xi-\operatorname{div}_{x}\left(\left|u_{1}-u_{2}\right|\left(\nu\left(\phi_{1}\right)+\nu\left(\phi_{2}\right)\right)\right) \xi\right. \\
& \left.\quad+\left|u_{1}-u_{2}\right|\left(\mathfrak{b}\left(\phi_{1}\right)-\beta\right) \xi+\operatorname{sign}\left(u_{1}-u_{2}\right)\left(\mathfrak{b}\left(\phi_{1}\right)-\mathfrak{b}\left(\phi_{2}\right)\right) u_{2} \xi\right) d x d a d t \\
& +\int_{0}^{\infty} \int_{\mathbb{R}^{2}}\left|u_{1,0}(x)-u_{2,0}(x)\right| \xi(0, a, x) d x d a \\
& \geq \int_{0}^{\infty} \int_{0}^{\infty} \int_{\mathbb{R}^{2}} \operatorname{sign}\left(u_{1}-u_{2}\right)\left(u_{2} \operatorname{div}_{x}\left(\nu\left(\phi_{1}\right)\right)-u_{1} \operatorname{div}_{x}\left(\nu\left(\phi_{2}\right)\right) \xi d x d a d t .\right.
\end{aligned}
$$


Proof. We double the variables and write

$$
\rho_{1}=\rho_{1}(t, a, x), \quad \rho_{2}=\rho_{2}(s, b, y), \quad \phi_{1}=\phi_{1}(t, x), \quad \phi_{2}=\phi_{2}(s, y) \quad u_{1}=u_{1}(t, x) \quad u_{2}=u_{2}(s, y)
$$

where $x=\left(x_{1}, x_{2}\right)$ and $y=\left(y_{1}, y_{2}\right)$.

Consider the test function

$$
\Xi_{n}(t, s, a, b, x, y)=\xi\left(\frac{t+s}{2}, \frac{a+b}{2}, \frac{x+y}{2}\right) \lambda_{n}\left(\frac{s-t}{2}\right) \lambda_{n}\left(\frac{b-a}{2}\right) \lambda_{n}\left(\frac{y_{1}-x_{1}}{2}\right) \lambda_{n}\left(\frac{y_{2}-x_{2}}{2}\right),
$$

where

$$
\lambda_{n}(u)=n \lambda(n u), \quad \lambda \in C^{\infty}(\mathbb{R}), \quad \lambda \geq 0, \quad\|\lambda\|_{L^{1}(\mathbb{R})}=1, \quad \operatorname{supp}(\lambda) \subset[-1,1] .
$$

To prove inequality (3.1) we follow the doubling of variables argument appearing in [2, Lemma 3.1], and use the regularity of $\mathfrak{p}$ and the $L^{\infty}$ bounds on $u_{1}, u_{2}$. Then we have only to verify $(3.2)$. We write (1.15) for $u_{1}(t, x)$ using $u_{2}(s, y)$ as a constant and integrate over $(s, y)$

$\iiint \iiint\left(\left|u_{1}-u_{2}\right| \partial_{t} \Xi_{n}-\operatorname{div}_{x}\left(\left|u_{1}-u_{2}\right| \nu\left(\phi_{1}\right)\right) \Xi_{n}+\operatorname{sign}\left(u_{1}-u_{2}\right)\left(\mathfrak{b}\left(\phi_{1}\right)-\beta\right) u_{1} \Xi_{n}\right) \mathrm{d} x \mathrm{~d} y \mathrm{~d} a \mathrm{~d} b \mathrm{~d} t \mathrm{~d} s$ $+\iiint \iint\left|u_{1,0}(x)-u_{2}\right| \Xi_{n}(0, s, a, b, x, y) \mathrm{d} x \mathrm{~d} y \mathrm{~d} a \mathrm{~d} b \mathrm{~d} s$

$$
\geq \iiint \iiint \operatorname{sign}\left(u_{1}-u_{2}\right) u_{2} \operatorname{div}_{x}\left(\nu\left(\phi_{1}\right)\right) \Xi_{n} \mathrm{~d} x \mathrm{~d} y \mathrm{~d} a \mathrm{~d} b \mathrm{~d} t \mathrm{~d} s
$$

and we write 1.15) for $u_{2}(s, y)$ using $u_{1}(t, x)$ as a constant and integrate over $(t, x)$

$$
\begin{aligned}
& \iiint \iiint\left(\left|u_{1}-u_{2}\right| \partial_{s} \Xi_{n}-\operatorname{div}_{y}\left(\left|u_{1}-u_{2}\right| \nu\left(\phi_{2}\right)\right) \Xi_{n}-\operatorname{sign}\left(u_{1}-u_{2}\right)\left(\mathfrak{b}\left(\phi_{2}\right)-\beta\right) u_{2} \Xi_{n}\right) \mathrm{d} x \mathrm{~d} y \mathrm{~d} a \mathrm{~d} b \mathrm{~d} t \mathrm{~d} s \\
& +\iiint \iint\left|u_{1}-u_{2,0}(y)\right| \Xi_{n}(t, 0, a, b, x, y) \mathrm{d} x \mathrm{~d} y \mathrm{~d} a \mathrm{~d} b \mathrm{~d} t \\
& \geq-\iiint \iiint \operatorname{sign}\left(u_{1}-u_{2}\right) u_{1} \operatorname{div}_{x}\left(\nu\left(\phi_{2}\right)\right) \Xi_{n} \mathrm{~d} x \mathrm{~d} y \mathrm{~d} a \mathrm{~d} b \mathrm{~d} t \mathrm{~d} s .
\end{aligned}
$$

Summing (3.3) and (3.4) we have

$$
\begin{aligned}
& \iiint \iiint\left(\left|u_{1}-u_{2}\right|\left(\partial_{t} \Xi_{n}+\partial_{s} \Xi_{n}\right)-\operatorname{div}_{x}\left(\left|u_{1}-u_{2}\right| \nu\left(\phi_{1}\right)\right) \Xi_{n}-\operatorname{div}_{y}\left(\left|u_{1}-u_{2}\right| \nu\left(\phi_{2}\right)\right) \Xi_{n}\right. \\
& \left.+\left|u_{1}-u_{2}\right|\left(\mathfrak{b}\left(\phi_{1}\right)-\beta\right) \Xi_{n}+\operatorname{sign}\left(u_{1}-u_{2}\right)\left(\mathfrak{b}\left(\phi_{1}\right)-\mathfrak{b}\left(\phi_{2}\right)\right) u_{2} \Xi_{n}\right) \mathrm{d} x \mathrm{~d} y \mathrm{~d} a \mathrm{~d} b \mathrm{~d} t \mathrm{~d} s \\
& +\iiint \iint\left|u_{1,0}(x)-u_{2}\right| \Xi_{n}(0, s, a, b, x, y) \mathrm{d} x \mathrm{~d} y \mathrm{~d} a \mathrm{~d} b \mathrm{~d} s \\
& +\iiint \iint\left|u_{1}-u_{2,0}(y)\right| \Xi_{n}(t, 0, a, b, x, y) \mathrm{d} x \mathrm{~d} y \mathrm{~d} a \mathrm{~d} b \mathrm{~d} t \\
& \quad \geq \iiint \iiint \operatorname{sign}\left(u_{1}-u_{2}\right) u_{2} \operatorname{div}_{x}\left(\nu\left(\phi_{1}\right)\right) \Xi_{n} \mathrm{~d} x \mathrm{~d} y \mathrm{~d} a \mathrm{~d} b \mathrm{~d} t \mathrm{~d} s \\
& \quad-\iiint \iiint \operatorname{sign}\left(u_{1}-u_{2}\right) u_{1} \operatorname{div}_{x}\left(\nu\left(\phi_{2}\right)\right) \Xi_{n} \mathrm{~d} x \mathrm{~d} y \mathrm{~d} a \mathrm{~d} b \mathrm{~d} t \mathrm{~d} s .
\end{aligned}
$$

As $n \rightarrow \infty$ we get 3.2 . 
Lemma 3.2. For every $t \geq 0$, the following inequality holds

$$
\begin{array}{r}
\left\|\phi_{1}(t, \cdot)-\phi_{2}(t, \cdot)\right\|_{L^{2}\left(\mathbb{R}^{2}\right)}^{2}+\mu e^{C t} \int_{0}^{t} \int_{\mathbb{R}^{2}} e^{-C s}\left|\nabla_{x}\left(\phi_{1}-\phi_{2}\right)\right|^{2} d x d s \leq C e^{C t}\left\|\rho_{1,0}-\rho_{2,0}\right\|_{L^{1}\left((0, \infty) \times \mathbb{R}^{2}\right)}^{2} \\
+C e^{C t} \int_{0}^{t} \int_{0}^{\infty} \int_{\mathbb{R}^{2}} e^{-C s}\left(\rho_{1}-\rho_{2}\right)^{2} d x d a d s+C e^{C t} \int_{0}^{t} \int_{\mathbb{R}^{2}} e^{-C s}\left(u_{1}-u_{2}\right)^{2} d x d s .
\end{array}
$$

In particular, we have that

ine:new (3.6)

$$
\begin{aligned}
& \left(\int_{0}^{t} \int_{\mathbb{R}^{2}} e^{-C s}\left|\nabla_{x}\left(\phi_{1}-\phi_{2}\right)\right|^{2} d x d s\right)^{1 / 2} \leq C e^{C t}\left\|\rho_{1,0}-\rho_{2,0}\right\|_{L^{1}\left((0, \infty) \times \mathbb{R}^{2}\right)} \\
& \quad+C e^{C t}\left(\int_{0}^{t}\left\|\rho_{1}(s, \cdot, \cdot)-\rho_{2}(s, \cdot, \cdot)\right\|_{L^{1}\left((0, \infty) \times \mathbb{R}^{2}\right)}^{2} d s\right)^{1 / 2}+C e^{C t}\left(\int_{0}^{t}\left\|u_{1}(s, \cdot)-u_{2}(s, \cdot)\right\|_{L^{1}\left(\mathbb{R}^{2}\right)}^{2} d s\right)^{1 / 2} .
\end{aligned}
$$

Proof. Since $\phi_{\varepsilon_{k}}$ satisfies

$$
\begin{aligned}
\partial_{t} \phi_{\varepsilon_{k}}-\mu \Delta_{x} \phi_{\varepsilon_{k}}+ & \operatorname{div}_{x}\left(\left(\int_{0}^{\infty} \rho_{\varepsilon_{k}} \chi_{1} \mathrm{~d} a\right) \mathbf{v} Y_{\theta}\left(\phi_{\varepsilon_{k}}-R\right)\right) \\
& =\int_{0}^{\infty} \rho_{\varepsilon_{k}} \chi_{2}\left(-\mathfrak{d}-\mathfrak{p}\left(a, u_{\varepsilon_{k}}\right)\right) \mathrm{d} a+\int_{0}^{\infty} \rho_{\varepsilon_{k}}\left(\left(1+\varepsilon \chi^{\prime}\right) \chi_{2}^{\prime}+\varepsilon \chi \chi_{2}^{\prime \prime}\right) \mathrm{d} a,
\end{aligned}
$$

as $k \rightarrow \infty$ we get the equation of $\phi$. Then, subtracting the equation for $\phi_{2}$ from the equation for $\phi_{1}$ we obtain

$$
\begin{aligned}
& \partial_{t}\left(\phi_{1}-\phi_{2}\right)-\mu \Delta_{x}\left(\phi_{1}-\phi_{2}\right)+\operatorname{div}_{x}\left(\left(\int_{0}^{\infty}\left(\rho_{1}-\rho_{2}\right) \chi_{1} \mathrm{~d} a\right) \mathbf{v} Y_{\theta}\left(\phi_{1}-R\right)\right) \\
& \quad+\operatorname{div}_{x}\left(\left(\int_{0}^{\infty} \rho_{2} \chi_{1} \mathrm{~d} a\right) \mathbf{v}\left(Y_{\theta}\left(\phi_{1}-R\right)-Y_{\theta}\left(\phi_{2}-R\right)\right)\right) \\
& =\int_{0}^{\infty}\left(\rho_{1}-\rho_{2}\right)\left(\chi_{2}^{\prime}-\mathfrak{d} \chi_{2}-\mathfrak{p}\left(a, u_{1}\right) \chi_{2}\right) \mathrm{d} a-\int_{0}^{\infty} \rho_{2}\left(\mathfrak{p}\left(a, u_{1}\right)-\mathfrak{p}\left(a, u_{2}\right)\right) \chi_{2} \mathrm{~d} a .
\end{aligned}
$$

Then

$$
\begin{aligned}
\frac{d}{d t} & \int_{\mathbb{R}^{2}} \frac{\left(\phi_{1}-\phi_{2}\right)^{2}}{2} \mathrm{~d} x+\mu \int_{\mathbb{R}^{2}}\left|\nabla_{x}\left(\phi_{1}-\phi_{2}\right)\right|^{2} \mathrm{~d} x \\
= & \int_{\mathbb{R}^{2}}\left(\phi_{1}-\phi_{2}\right)\left(\int_{0}^{\infty}\left(\rho_{1}-\rho_{2}\right)\left(\chi_{2}^{\prime}-\mathfrak{d} \chi_{2}-\mathfrak{p}\left(a, u_{1}\right) \chi_{2}\right) \mathrm{d} a\right) \mathrm{d} x \\
& -\int_{\mathbb{R}^{2}}\left(\phi_{1}-\phi_{2}\right)(\int_{0}^{\infty} \rho_{2} \underbrace{\left(\mathfrak{p}\left(a, u_{1}\right)-\mathfrak{p}\left(a, u_{2}\right)\right)}_{\leq c\left|u_{1}-u_{2}\right|} \chi_{2} \mathrm{~d} a) \mathrm{d} x \\
& -\int_{\mathbb{R}^{2}}\left(\phi_{1}-\phi_{2}\right) \operatorname{div}_{x}\left(\left(\int_{0}^{\infty}\left(\rho_{1}-\rho_{2}\right) \chi_{1} \mathrm{~d} a\right) \mathbf{v} Y_{\theta}\left(\phi_{1}-R\right)\right) \mathrm{d} x \\
& -\int_{\mathbb{R}^{2}}\left(\phi_{1}-\phi_{2}\right) \operatorname{div} x\left(\left(\int_{0}^{\infty} \rho_{2} \chi_{1} \mathrm{~d} a\right) \mathbf{v}\left(Y_{\theta}\left(\phi_{1}-R\right)-Y_{\theta}\left(\phi_{2}-R\right)\right)\right) \mathrm{d} x \\
\leq & c \int_{\mathbb{R}^{2}}\left(\phi_{1}-\phi_{2}\right)^{2} \mathrm{~d} x+c e^{c t} \int_{\mathbb{R}^{2}}\left(u_{1}-u_{2}\right)^{2} \mathrm{~d} x+c e^{c t} \int_{0}^{\infty} \int_{\mathbb{R}^{2}}\left(\rho_{1}-\rho_{2}\right)^{2} \mathrm{~d} x \mathrm{~d} a \\
& +\frac{\mu}{2} \int_{\mathbb{R}^{2}}\left|\nabla_{x}\left(\phi_{1}-\phi_{2}\right)\right|^{2} \mathrm{~d} x .
\end{aligned}
$$

Using Gronwall Lemma we get

$$
\begin{aligned}
& \left\|\phi_{1}(t, \cdot)-\phi_{2}(t, \cdot)\right\|_{L^{2}\left(\mathbb{R}^{2}\right)}^{2}+\mu e^{c t} \int_{0}^{t} \int_{\mathbb{R}^{2}} e^{-c s}\left|\nabla_{x}\left(\phi_{1}-\phi_{2}\right)\right|^{2} \mathrm{~d} x \mathrm{~d} s \\
& \leq e^{c t}\left\|\phi_{1,0}-\phi_{2,0}\right\|_{L^{2}\left(\mathbb{R}^{2}\right)}^{2}+c e^{c t} \int_{0}^{t} \int_{0}^{\infty} \int_{\mathbb{R}^{2}} e^{-c s}\left(\rho_{1}-\rho_{2}\right)^{2} \mathrm{~d} x \mathrm{~d} a \mathrm{~d} s+c e^{c t} \int_{0}^{t} \int_{\mathbb{R}^{2}} e^{-c s}\left(u_{1}-u_{2}\right)^{2} \mathrm{~d} x \mathrm{~d} s .
\end{aligned}
$$


Finally, we obtain (3.5) and (3.6) from the definition of $\phi_{1}$ and $\phi_{2}$.

We are now ready to complete the proof of Theorem 1.1 .

Proof. Our goal is to prove the inequality 1.16$)$. We rewrite $(3.1)$ and $(3.2)$ as

$$
\begin{aligned}
& \int_{0}^{\infty} \int_{0}^{\infty} \int_{\mathbb{R}^{2}}\left(\left|\rho_{1}-\rho_{2}\right|\left(\partial_{t} \xi_{n}+\partial_{a} \xi_{n}\right)-\left|\rho_{1}-\rho_{2}\right| \chi_{1}\left(Y_{\theta}\left(\phi_{1}-R\right)+Y_{\theta}\left(\phi_{2}-R\right)\right) \mathbf{v} \cdot \nabla_{x} \xi_{n}\right. \\
& \quad+\mu\left|\rho_{1}-\rho_{2}\right| \Delta_{x} \xi_{n}-\left|\rho_{1}-\rho_{2}\right| \mathfrak{d} \xi_{n}-\left|\rho_{1}-\rho_{2}\right| \mathfrak{p}\left(a, u_{1}\right) \xi_{n} \\
& \left.\quad-\operatorname{sign}\left(\rho_{1}-\rho_{2}\right)\left(\mathfrak{p}\left(a, u_{1}\right)-\mathfrak{p}\left(a, u_{2}\right)\right) \rho_{2} \xi_{n}\right) \mathrm{d} x \mathrm{~d} a \mathrm{~d} t \\
& +\int_{0}^{\infty} \int_{\mathbb{R}^{2}}\left|\rho_{1}\left(t, 0^{+}, x\right)-\rho_{2}\left(t, 0^{+}, x\right)\right| \xi_{n}(t, 0, x) \mathrm{d} x \mathrm{~d} t \\
& +\int_{0}^{\infty} \int_{\mathbb{R}^{2}}\left|\rho_{1,0}(a, x)-\rho_{2,0}(a, x)\right| \xi_{n}(0, a, x) \mathrm{d} x \mathrm{~d} a \\
& \geq \int_{0}^{\infty} \int_{0}^{\infty} \int_{\mathbb{R}^{2}} \operatorname{sign}\left(\rho_{1}-\rho_{2}\right) \chi_{1}\left(\rho_{2} \operatorname{div}_{x}\left(\mathbf{v} Y_{\theta}\left(\phi_{1}-R\right)\right)-\rho_{1} \operatorname{div}_{x}\left(\mathbf{v}(x) Y_{\theta}\left(\phi_{2}-R\right)\right)\right) \xi_{n} \mathrm{~d} x \mathrm{~d} a \mathrm{~d} t,
\end{aligned}
$$

and

$$
\begin{aligned}
& \int_{0}^{\infty} \int_{0}^{\infty} \int_{\mathbb{R}^{2}}\left(\left|u_{1}-u_{2}\right| \partial_{t} \xi_{n}+\left|u_{1}-u_{2}\right|\left(\nu\left(\phi_{1}\right)+\nu\left(\phi_{2}\right)\right) \cdot \nabla_{x} \xi_{n}+\left|u_{1}-u_{2}\right|\left(\mathfrak{b}\left(\phi_{1}\right)-\beta\right) \xi_{n}\right. \\
& \left.\quad+\operatorname{sign}\left(u_{1}-u_{2}\right)\left(\mathfrak{b}\left(\phi_{1}\right)-\mathfrak{b}\left(\phi_{2}\right)\right) u_{2} \xi_{n}\right) \mathrm{d} x \mathrm{~d} a \mathrm{~d} t \\
& +\int_{0}^{\infty} \int_{\mathbb{R}^{2}}\left|u_{1,0}(x)-u_{2,0}(x)\right| \xi_{n}(0, a, x) \mathrm{d} x \mathrm{~d} a \\
& \geq \int_{0}^{\infty} \int_{0}^{\infty} \int_{\mathbb{R}^{2}} \operatorname{sign}\left(u_{1}-u_{2}\right)\left(u_{2} \operatorname{div}_{x}\left(\nu\left(\phi_{1}\right)\right)-u_{1} \operatorname{div}_{x}\left(\nu\left(\phi_{2}\right)\right)\right) \xi_{n} \mathrm{~d} x \mathrm{~d} a \mathrm{~d} t,
\end{aligned}
$$

where $\left\{\xi_{n}\right\}_{n}$ is a sequence of nonnegative test functions approximating the characteristic function of the strip $(-\infty, t) \times \mathbb{R} \times \mathbb{R}^{2}$. Sending $n \rightarrow \infty$, we have that

$$
\begin{aligned}
& \left\|u_{1}(t, \cdot)-u_{2}(t, \cdot)\right\|_{L^{1}\left(\mathbb{R}^{2}\right)} \\
& \leq\left\|u_{1,0}-u_{2,0}\right\|_{L^{1}\left(\mathbb{R}^{2}\right)}-\int_{0}^{t} \int_{\mathbb{R}^{2}} \operatorname{sign}\left(u_{1}-u_{2}\right)\left(u_{2} \operatorname{div}_{x}\left(\nu\left(\phi_{1}\right)\right)-u_{1} \operatorname{div}_{x}\left(\nu\left(\phi_{2}\right)\right)\right) \mathrm{d} x \mathrm{~d} s \\
& \quad+\int_{0}^{t} \int_{\mathbb{R}^{2}}\left|u_{1}-u_{2}\right| \underbrace{\mathfrak{b}\left(\phi_{1}\right)}_{\leq c}+\operatorname{sign}\left(u_{1}-u_{2}\right) \underbrace{\left(\mathfrak{b}\left(\phi_{1}\right)-\mathfrak{b}\left(\phi_{2}\right)\right)}_{\leq c\left|\phi_{1}-\phi_{2}\right|} u_{2} \mathrm{~d} x \mathrm{~d} s \\
& \leq\left\|u_{1,0}-u_{2,0}\right\|_{L^{1}\left(\mathbb{R}^{2}\right)}+\int_{0}^{t} \int_{\mathbb{R}^{2}}\left|u_{1}-u_{2}\right| \operatorname{div}_{x}\left(\nu\left(\phi_{1}\right)\right) \mathrm{d} x \mathrm{~d} s \\
& \quad-\int_{0}^{t} \int_{\mathbb{R}^{2}} \operatorname{sign}\left(u_{1}-u_{2}\right) u_{1} \underbrace{\operatorname{div}\left(\nu\left(\phi_{1}\right)-\nu\left(\phi_{2}\right)\right)}_{\operatorname{see}(2.10)} \mathrm{d} x \mathrm{~d} s \\
& \quad+c \int_{0}^{t} \int_{\mathbb{R}^{2}}\left|u_{1}-u_{2}\right| \mathrm{d} x \mathrm{~d} s+c e^{c t} \int_{0}^{t} \int_{\mathbb{R}^{2}}\left|\phi_{1}-\phi_{2}\right| \mathrm{d} x \mathrm{~d} s \\
& \leq\left\|u_{1,0}-u_{2,0}\right\|_{L^{1}\left(\mathbb{R}^{2}\right)}+c e^{c t} \int_{0}^{t} \int_{\mathbb{R}^{2}}^{\left|u_{1}-u_{2}\right| \mathrm{d} x \mathrm{~d} s} \\
& \quad+c e^{c t} \int_{0}^{t}\left(\int_{\mathbb{R}^{2}}\left|\operatorname{div}_{x}\left(\nu\left(\phi_{1}\right)-\nu\left(\phi_{2}\right)\right)\right|^{2} \mathrm{~d} x\right)^{1 / 2} \mathrm{~d} s \\
& \quad+c \int_{0}^{t} \int_{\mathbb{R}^{2}}\left|u_{1}-u_{2}\right| \mathrm{d} x \mathrm{~d} s+c e^{c t} \int_{0}^{t} \int_{\mathbb{R}^{2}}\left|\phi_{1}-\phi_{2}\right| \mathrm{d} x \mathrm{~d} s \\
& \leq\left\|u_{1,0}-u_{2,0}\right\|_{L^{1}\left(\mathbb{R}^{2}\right)}+c\left(1+e^{c t}\right) \int_{0}^{t}\left\|u_{1}(s, \cdot)-u_{2}(s, \cdot)\right\|_{L^{1}\left(\mathbb{R}^{2}\right)} \mathrm{d} s
\end{aligned}
$$




$$
+c e^{c t} \int_{0}^{t}\left\|\rho_{1}(s, \cdot, \cdot)-\rho_{2}(s, \cdot, \cdot)\right\|_{L^{1}\left((0, \infty) \times \mathbb{R}^{2}\right)} \mathrm{d} s,
$$

and

$$
\begin{aligned}
& \left\|\rho_{1}(t, \cdot, \cdot)-\rho_{2}(t, \cdot, \cdot)\right\|_{L^{1}\left((0, \infty) \times \mathbb{R}^{2}\right)} \\
& \leq\left\|\rho_{1,0}-\rho_{2,0}\right\|_{L^{1}\left((0, \infty) \times \mathbb{R}^{2}\right)}+\int_{0}^{t} \int_{\mathbb{R}^{2}}\left|\rho_{1}\left(s, 0^{+}, x\right)-\rho_{2}\left(s, 0^{+}, x\right)\right| \mathrm{d} x \mathrm{~d} s \\
& -\int_{0}^{t} \int_{0}^{\infty} \int_{\mathbb{R}^{2}} \operatorname{sign}\left(\rho_{1}-\rho_{2}\right) \chi_{1}(a)\left(\rho_{2} \operatorname{div}_{x}\left(\mathbf{v} Y_{\theta}\left(\phi_{1}-R\right)\right)-\rho_{1} \operatorname{div}_{x}\left(\mathbf{v} Y_{\theta}\left(\phi_{2}-R\right)\right)\right) \mathrm{d} x \mathrm{~d} a \mathrm{~d} s \\
& -\int_{0}^{t} \int_{0}^{\infty} \int_{\mathbb{R}^{2}} \operatorname{sign}\left(\rho_{1}-\rho_{2}\right)\left(\mathfrak{p}\left(a, u_{1}\right)-\mathfrak{p}\left(a, u_{2}\right)\right) \rho_{2} \mathrm{~d} x \mathrm{~d} a \mathrm{~d} s \\
& \leq\left\|\rho_{1,0}-\rho_{2,0}\right\|_{L^{1}\left((0, \infty) \times \mathbb{R}^{2}\right)}+\int_{0}^{t} \int_{0}^{\infty} \int_{\mathbb{R}^{2}}\left|\mathcal{A}\left(\phi_{1}\right) \rho_{1}-\mathcal{A}\left(\phi_{2}\right) \rho_{2}\right| \chi_{3} \omega \mathrm{d} x \mathrm{~d} s \\
& -\int_{0}^{t} \int_{0}^{\infty} \int_{\mathbb{R}^{2}} \operatorname{sign}\left(\rho_{1}-\rho_{2}\right) \chi_{1}(a)\left(\rho_{2} \operatorname{div}_{x}\left(\mathbf{v} Y_{\theta}\left(\phi_{1}-R\right)\right)-\rho_{1} \operatorname{div}_{x}\left(\mathbf{v} Y_{\theta}\left(\phi_{2}-R\right)\right)\right) \mathrm{d} x \mathrm{~d} a \mathrm{~d} s \\
& -\int_{0}^{t} \int_{0}^{\infty} \int_{\mathbb{R}^{2}} \operatorname{sign}\left(\rho_{1}-\rho_{2}\right)\left(\mathfrak{p}\left(a, u_{1}\right)-\mathfrak{p}\left(a, u_{2}\right)\right) \rho_{2} \mathrm{~d} x \mathrm{~d} a \mathrm{~d} s \\
& \leq\left\|\rho_{1,0}-\rho_{2,0}\right\|_{L^{1}\left((0, \infty) \times \mathbb{R}^{2}\right)}+\int_{0}^{t} \int_{0}^{\infty} \int_{\mathbb{R}^{2}} \mathcal{A}\left(\phi_{1}\right)\left|\rho_{1}-\rho_{2}\right| \chi_{3} \omega \mathrm{d} x \mathrm{~d} a \mathrm{~d} s \\
& +\int_{0}^{t} \int_{0}^{\infty} \int_{\mathbb{R}^{2}}\left|\mathcal{A}\left(\phi_{1}\right)-\mathcal{A}\left(\phi_{2}\right)\right| \rho_{2} \chi_{3} \omega \mathrm{d} x \mathrm{~d} a \mathrm{~d} s \\
& +\int_{0}^{t} \int_{0}^{\infty} \int_{\mathbb{R}^{2}}\left|\rho_{1}-\rho_{2}\right| \chi_{1}(a) \operatorname{div}_{x}\left(\mathbf{v} Y_{\theta}\left(\phi_{1}-R\right)\right) \mathrm{d} x \mathrm{~d} a \mathrm{~d} s \\
& -\int_{0}^{t} \int_{0}^{\infty} \int_{\mathbb{R}^{2}} \operatorname{sign}\left(\rho_{1}-\rho_{2}\right) \chi_{1}(a) \rho_{1} \operatorname{div}_{x}(\mathbf{v})\left(Y_{\theta}\left(\phi_{1}-R\right)-Y_{\theta}\left(\phi_{2}-R\right)\right) \mathrm{d} x \mathrm{~d} a \mathrm{~d} s \\
& -\int_{0}^{t} \int_{0}^{\infty} \int_{\mathbb{R}^{2}} \operatorname{sign}\left(\rho_{1}-\rho_{2}\right) \chi_{1}(a) \rho_{1} \mathbf{v} \cdot \nabla_{x}\left(Y_{\theta}\left(\phi_{1}-R\right)-Y_{\theta}\left(\phi_{2}-R\right)\right) \mathrm{d} x \mathrm{~d} a \mathrm{~d} s \\
& +c e^{c t} \int_{0}^{t}\left\|u_{1}(s, \cdot)-u_{2}(s, \cdot)\right\|_{L^{1}\left(\mathbb{R}^{2}\right)} \mathrm{d} s \\
& \leq\left\|\rho_{1,0}-\rho_{2,0}\right\|_{L^{1}\left((0, \infty) \times \mathbb{R}^{2}\right)}+c \int_{0}^{t}\left\|\rho_{1}(s, \cdot, \cdot)-\rho_{2}(s, \cdot, \cdot)\right\|_{L^{1}\left((0, \infty) \times \mathbb{R}^{2}\right)} \mathrm{d} s \\
& +c e^{c t} \int_{0}^{t} \int_{\mathbb{R}^{2}}\left|\mathcal{A}\left(\phi_{1}\right)-\mathcal{A}\left(\phi_{2}\right)\right| \mathrm{d} x \mathrm{~d} s \\
& +c e^{c t} \int_{0}^{t} \int_{\mathbb{R}^{2}}\left|Y_{\theta}\left(\phi_{1}-R\right)-Y_{\theta}\left(\phi_{2}-R\right)\right| \mathrm{d} x \mathrm{~d} s \\
& +c e^{c t}\left(\int_{0}^{t} \int_{\mathbb{R}^{2}}\left|\nabla_{x}\left(Y_{\theta}\left(\phi_{1}-R\right)-Y_{\theta}\left(\phi_{2}-R\right)\right)\right|^{2} \mathrm{~d} x \mathrm{~d} s\right)^{1 / 2} \\
& +c e^{c t} \int_{0}^{t}\left\|u_{1}(s, \cdot)-u_{2}(s, \cdot)\right\|_{L^{1}\left(\mathbb{R}^{2}\right)} \mathrm{d} s \\
& \leq\left\|\rho_{1,0}-\rho_{2,0}\right\|_{L^{1}\left((0, \infty) \times \mathbb{R}^{2}\right)}+c\left(1+e^{c t}\right) \int_{0}^{t}\left\|\rho_{1}(s, \cdot, \cdot)-\rho_{2}(s, \cdot, \cdot)\right\|_{L^{1}\left((0, \infty) \times \mathbb{R}^{2}\right)} \mathrm{d} s \\
& +c e^{c t}\left(\int_{0}^{t} \int_{\mathbb{R}^{2}}\left|\nabla_{x}\left(\phi_{1}-\phi_{2}\right)\right|^{2} \mathrm{~d} x \mathrm{~d} s\right)^{1 / 2}+c e^{c t} \int_{0}^{t}\left\|u_{1}(s, \cdot)-u_{2}(s, \cdot)\right\|_{L^{1}\left(\mathbb{R}^{2}\right)} \mathrm{d} s .
\end{aligned}
$$

Using (3.6), we have

$$
\left\|u_{1}(t, \cdot)-u_{2}(t, \cdot)\right\|_{L^{1}\left(\mathbb{R}^{2}\right)}^{2}+\left\|\rho_{1}(t, \cdot, \cdot)-\rho_{2}(t, \cdot, \cdot)\right\|_{L^{1}\left((0, \infty) \times \mathbb{R}^{2}\right)}^{2}
$$




$$
\begin{aligned}
& \leq\left\|u_{1,0}-u_{2,0}\right\|_{L^{1}\left(\mathbb{R}^{2}\right)}^{2}+c\left(1+e^{c t}\right) \int_{0}^{t}\left\|u_{1}(s, \cdot)-u_{2}(s, \cdot)\right\|_{L^{1}\left(\mathbb{R}^{2}\right)}^{2} \mathrm{~d} s \\
& +c\left(1+e^{c t}\right)\left\|\rho_{1,0}-\rho_{2,0}\right\|_{L^{1}\left((0, \infty) \times \mathbb{R}^{2}\right)}^{2}+c\left(1+e^{c t}\right) \int_{0}^{t}\left\|\rho_{1}(s, \cdot, \cdot)-\rho_{2}(s, \cdot, \cdot)\right\|_{L^{1}\left((0, \infty) \times \mathbb{R}^{2}\right)}^{2} \mathrm{~d} s .
\end{aligned}
$$

Finally, we use the Gronwall Lemma to obtain the result 1.16).

\section{Proof of Lemma 2.1}

Proof. This lemma is similar to [4, Lemma 4.1], in particular, (2.5) and (2.8) already appeared there. For completeness we sketch the proof of the other estimates.

(2.6). directly comes from the definition of $\nu$ in $(1.2)$ and the expression of $\operatorname{div}_{x}(\nu(\phi))$ in $(2.38)$.

(2.7). We compute $\nabla_{x} \nu(\phi)$ by using the fact that $\nabla_{x}(f \nu)=f \nabla_{x} \nu+\nu \otimes \nabla_{x} f$

$$
\begin{aligned}
\nabla_{x} \nu(\phi) & =\kappa \frac{1}{\left(1+\left\|\phi * \nabla_{x} \eta\right\|^{2}\right)^{1 / 2}} \nabla_{x}\left(\phi * \nabla_{x} \eta\right)+\kappa\left(\phi * \nabla_{x} \eta\right) \otimes \nabla_{x} \frac{1}{\left(1+\left\|\phi * \nabla_{x} \eta\right\|^{2}\right)^{1 / 2}} \\
& =\kappa \frac{\phi * \nabla_{x}^{2} \eta}{\left(1+\left\|\phi * \nabla_{x} \eta\right\|^{2}\right)^{1 / 2}}-\kappa\left(\phi * \nabla_{x} \eta\right) \otimes \frac{\left(\phi * \nabla_{x}^{2} \eta\right)\left(\phi * \nabla_{x} \eta\right)}{\left(1+\left\|\phi * \nabla_{x} \eta\right\|^{2}\right)^{3 / 2}}
\end{aligned}
$$

then (notice that to shorten the notations we write $L^{\infty}$ for $L^{\infty}\left(\mathbb{R}^{2} ; \mathbb{R}^{2 \times 2}\right.$ )

$$
\begin{aligned}
& \left\|\nabla_{x} \nu(\phi)\right\|_{L^{\infty}} \\
& \leq \kappa \frac{\left\|\phi * \nabla_{x}^{2} \eta\right\|_{L^{\infty}}}{\left(1+\left\|\phi * \nabla_{x} \eta\right\|^{2}\right)^{1 / 2}}+\kappa\left\|\frac{\phi * \nabla_{x} \eta}{\left(1+\left\|\phi * \nabla_{x} \eta\right\|^{2}\right)^{1 / 2}} \otimes \frac{\phi * \nabla_{x}^{2} \eta}{\left(1+\left\|\phi * \nabla_{x} \eta\right\|^{2}\right)^{1 / 2}} \frac{\phi * \nabla_{x} \eta}{\left(1+\left\|\phi * \nabla_{x} \eta\right\|^{2}\right)^{1 / 2}}\right\|_{L^{\infty}} \\
& \leq \kappa\left\|\phi * \nabla_{x}^{2} \eta\right\|_{L^{\infty}}+\kappa\left\|\frac{\phi * \nabla_{x} \eta}{\left(1+\left\|\phi * \nabla_{x} \eta\right\|^{2}\right)^{1 / 2}}\right\|_{L^{\infty}}\left\|\phi * \nabla_{x}^{2} \eta\right\|_{L^{\infty}}\left\|\frac{\phi * \nabla_{x} \eta}{\left(1+\left\|\phi * \nabla_{x} \eta\right\|^{2}\right)^{1 / 2}}\right\|_{L^{\infty}} \\
& \leq 2 \kappa\left\|\phi * \nabla_{x}^{2} \eta\right\|_{L^{\infty}} \leq 2 \kappa\left\|\nabla_{x}^{2} \eta\right\|_{L^{\infty}}\|\phi\|_{L^{1}\left(\mathbb{R}^{2} ; \mathbb{R}\right)} .
\end{aligned}
$$

(2.9). We compute gradient of 2.38)

$$
\nabla_{x} \operatorname{div}_{x}(\nu(\phi))=\kappa \frac{\phi * \nabla \Delta_{x} \eta}{\left(1+\left\|\phi * \nabla_{x} \eta\right\|^{2}\right)^{3 / 2}}-3 \kappa\left(\phi * \Delta_{x} \eta\right) \frac{\phi * \nabla_{x}^{2} \eta}{\left(1+\left\|\phi * \nabla_{x} \eta\right\|^{2}\right)^{2}} \frac{\phi * \nabla_{x} \eta}{\left(1+\left\|\phi * \nabla_{x} \eta\right\|^{2}\right)^{1 / 2}}
$$

then

$$
\begin{aligned}
& \left\|\nabla_{x} \operatorname{div}_{x}(\nu(\phi))\right\|_{L^{2}\left(\mathbb{R}^{2} ; \mathbb{R}^{2}\right)} \\
& \leq \kappa\left\|\phi * \nabla \Delta_{x} \eta\right\|_{L^{2}\left(\mathbb{R}^{2} ; \mathbb{R}^{2}\right)}+3 \kappa\left\|\phi * \Delta_{x} \eta\right\|_{L^{2}\left(\mathbb{R}^{2} ; \mathbb{R}^{2}\right)}\left\|\phi * \nabla_{x}^{2} \eta\right\|_{L^{\infty}\left(\mathbb{R}^{2} ; \mathbb{R}^{2 \times 2}\right)} \\
& \leq \kappa\|\phi\|_{L^{1}\left(\mathbb{R}^{2} ; \mathbb{R}\right)}\left(\left\|\nabla \Delta_{x} \eta\right\|_{L^{2}\left(\mathbb{R}^{2} ; \mathbb{R}^{2}\right)}+3\left\|\Delta_{x} \eta\right\|_{L^{2}\left(\mathbb{R}^{2} ; \mathbb{R}\right)}\left\|\nabla_{x}^{2} \eta\right\|_{L^{\infty}\left(\mathbb{R}^{2} ; \mathbb{R}^{2 \times 2}\right)}\|\phi\|_{L^{1}\left(\mathbb{R}^{2} ; \mathbb{R}\right)}\right) .
\end{aligned}
$$

(2.10). We have that

$$
\begin{aligned}
\operatorname{div}_{x}\left(\nu\left(\phi_{1}\right)-\nu\left(\phi_{2}\right)\right) \\
\quad=\kappa \frac{\left(\phi_{1}-\phi_{2}\right) * \Delta_{x} \eta}{\left(1+\left\|\phi_{1} * \nabla_{x} \eta\right\|^{2}\right)^{3 / 2}}+\kappa\left(\phi_{2} * \Delta_{x} \eta\right)\left(\frac{1}{\left(1+\left\|\phi_{1} * \nabla_{x} \eta\right\|^{2}\right)^{3 / 2}}-\frac{1}{\left(1+\left\|\phi_{2} * \nabla_{x} \eta\right\|^{2}\right)^{3 / 2}}\right) .
\end{aligned}
$$

Using the inequality $\left|\left(1+x^{2}\right)^{-3 / 2}-\left(1+y^{2}\right)^{-3 / 2}\right| \leq \frac{48}{25 \sqrt{5}}|x-y|$, we obtain

$$
\left\|\operatorname{div}_{x}\left(\nu\left(\phi_{1}\right)-\nu\left(\phi_{2}\right)\right)\right\|_{L^{2}\left(\mathbb{R}^{2} ; \mathbb{R}\right)}
$$




$$
\begin{aligned}
& \leq \kappa\left\|\left(\phi_{1}-\phi_{2}\right) * \Delta_{x} \eta\right\|_{L^{2}\left(\mathbb{R}^{2} ; \mathbb{R}\right)}+\frac{48}{25 \sqrt{5}} \kappa\left\|\phi_{2} * \Delta_{x} \eta\right\|_{L^{\infty}\left(\mathbb{R}^{2} ; \mathbb{R}\right)}\left\|\left(\phi_{1}-\phi_{2}\right) * \nabla_{x} \eta\right\|_{L^{2}\left(\mathbb{R}^{2} ; \mathbb{R}^{2}\right)} \\
& \leq \kappa\left\|\phi_{1}-\phi_{2}\right\|_{L^{1}\left(\mathbb{R}^{2}, \mathbb{R}\right)}\left(\left\|\Delta_{x} \eta\right\|_{L^{2}\left(\mathbb{R}^{2} ; \mathbb{R}\right)}+\frac{48}{25 \sqrt{5}}\left\|\nabla_{x} \eta\right\|_{L^{2}\left(\mathbb{R}^{2}, \mathbb{R}^{2}\right)}\left\|\Delta_{x} \eta\right\|_{L^{\infty}\left(\mathbb{R}^{2} ; \mathbb{R}\right)}\left\|\phi_{2}\right\|_{L^{1}\left(\mathbb{R}^{2}, \mathbb{R}\right)}\right) .
\end{aligned}
$$

\section{Acknowledgments}

The second and the third author acknowledge the support of the Région Bourgogne FrancheComté, projet 2017-2020 "Analyse mathématique et simulation numérique d'EDP issus de problèmes de contrôle et du trafic routier".

The first author is member of the Gruppo Nazionale per l'Analisi Matematica, la Probabilità e le loro Applicazioni (GNAMPA) of the Istituto Nazionale di Alta Matematica (INdAM). He has been partially supported by the Research Project of National Relevance "Multiscale Innovative Materials and Structures" granted by the Italian Ministry of Education, University and Research (MIUR Prin 2017, project code 2017J4EAYB and the Italian Ministry of Education, University and Research under the Programme Department of Excellence Legge 232/2016 (Grant No. CUP - D94I18000260001).

\section{REFERENCES}

[1] K. Berthier, S. Piry, J.-F. Cosson, P. Giraudoux, J.-C. Foltête, R. Defaut, D. Truchetet, and X. Lambin. Dispersal, landscape and travelling waves in cyclic vole populations. Ecology letters, 17, 112013.

[2] G. M. Coclite, C. Donadello, and T. N. T. Nguyen. A PDE model for the spatial dynamics of a voles population structured in age. Nonlinear Anal., 196:111805, 26, 2020.

[3] R. M. Colombo and M. Garavello. Stability and optimization in structured population models on graphs. Mathematical Biosciences \& Engineering, 12:311-335, 2015.

[4] R. M. Colombo and E. Rossi. Hyperbolic predators vs. parabolic prey. Communications in Mathematical Sciences, 13(2):369 $-400,2015$.

[5] P. Delattre and P. Giraudoux. Le campagnol terrestre: prévention et contrôle des populations. QUAE, 2009.

[6] P. Giraudoux, P. Delattre, M. Habert, J. Quéré, S. Deblay, R. D. R. Defaut, M. Moissenet, D. Salvi, and D. Truchetet. Population dynamics of fosserial water vole (arvicola terrestris scherman) : a land use and a landscape perspective. Agriculture, Ecosystems and Environment, 66(1):47-60, 1997.

[7] P. Giraudoux, P. Villette, J. Quéré, and et al. Weather influences m. arvalis reproduction but not population dynamics in a 17-year time series. Sci Rep, 9:13942, 2019.

[8] G. Halliez, F. Renault, E. Vannard, G. Farny, S. Lavorel, and P. Giraudoux. Historical agricultural changes and the expansion of a water vole population in an alpine valley. Agriculture Ecosystems \& Environment, 212:198-206, 122015.

[9] F. Murat. L'injection du cône positif de $H^{-1}$ dans $W^{-1, q}$ est compacte pour tout $q<2$. J. Math. Pures Appl. (9), 60(3):309$322,1981$.

[10] J. D. Murray. Mathematical Biology I. An Introduction, volume 17 of Interdisciplinary Applied Mathematics. Springer, New York, 3 edition, 2002.

[11] E. Y. Panov. Existence and strong pre-compactness properties for entropy solutions of a first-order quasilinear equation with discontinuous flux. Archive for Rational Mechanics and Analysis, 195(2):643-673, 2009.

[12] F. Saucy and S. Beat. Juvenile dispersal in the vole arvicola terrestris during rainy nights: A preliminary report. Bulletin de la Societe Vaudoise des Sciences Naturelles, 84:333-345, 121997.

[13] G. F. Webb. Population Models Structured by Age, Size, and Spatial Position, pages 1-49. Springer Berlin Heidelberg, Berlin, Heidelberg, 2008. 
(Giuseppe Maria Coclite)

Department of Mechanics, Mathematics and Management, Polytechnic University of Bari, Via E. Orabona 4, 70125 BARI, ITALY

Email address: giuseppemaria.coclite@poliba.it

(Carlotta Donadello and Thi Nhu Thao Nguyen)

Laboratoire de Mathématiques CNRS UMr6623, Université de Bourgogne Franche-Comté, 16 route de Gray, 25030 Besançon Cedex, France.

Email address: carlotta.donadello@univ-fcomte.fr, thi_nhu_thao.nguyen@univ-fcomte.fr 